\title{
K-STABILITY OF SMOOTH DEL PEZZO SURFACES
}

\author{
JIHUN PARK AND JOONYEONG WON
}

\begin{abstract}
In a new algebro-geometric way we completely determine whether smooth del Pezzo surfaces are K-(semi)stable or not.
\end{abstract}

In the present article, all varieties are defined over an algebraically closed field $k$ of characteristic 0 .

\section{INTRODUCTION}

Since entering the 21st century we have witnessed dramatic developments in the study of the Yau-Tian-Donaldson conjecture concerning the existence of Kähler-Einstein metrics on Fano manifolds and stability. The challenge to the conjecture has been highlighted by Chen, Donaldson, Sun and Tian who have completed the proof of the following celebrated statement ( [6], 7], [8], 25]).

Theorem 1.1. Let $X$ be a smooth Fano variety defined over $\mathbb{C}$. It admits a Kähler-Einstein metric if and only if the pair $\left(X,-K_{X}\right)$ is K-polystable.

The motivation of this article cannot be expressed in a better way than quoting the following phrase in one of the three articles by Chen, Donaldson and Sun ( [8]):

"On the other hand, we should point out that as things stand at present the result is of very limited use in concrete cases, so that there is no manifold $X$ known to us, not covered by other existence results and where we can deduce that $X$ has a Kähler-Einstein metric. This is because it seems a very difficult matter to test K-stability by a direct study of all possible degenerations. However, we are optimistic that this situation will change in the future, with a deeper analysis of the stability condition."

There are not so many results concerning K-stability of specified smooth Fano varieties, not deduced by Kähler-Einstein metrics. It seems almost infeasible to consider all possible degenerations of a given Fano manifold. Even for del Pezzo surfaces, we do not have complete description of their degenerations. There are a classification only for the projective plane and some partial classifications for the others ( [18, [19], 14], 22]). We also have a classification of del Pezzo surfaces with quotient singularities and Picard rank one that admit $\mathbb{Q}$-Gorenstein smoothings ( [14]). These are however not enough to directly test K-stability of del Pezzo surfaces.

Meanwhile, since asymptotic Chow-stability implies K-semistability (for instance, see [23]), an algebro-geometric proof for the K-semistability of the projective spaces can be yielded by the celebrated result of Kempf in [16] that a homogeneous rational variety embedded with a complete linear system is Chow-stable.

There are a few of algebro-geometric methods known to us that can be utilized to prove Kstability in concrete cases. One of the ways is based on the $\alpha$-invariant originally introduced by Tian ( [24]). The original definition of the $\alpha$-invariant was given in an analytic way. However,

This work has been supported by IBS-R003-D1, Institute for Basic Science in Korea. The authors are grateful to Kento Fujita and Yuji Odaka who brought the article 13] to their attention. They also thank Giulio Codogni who informed them of the article [16]. 
there is an algebro-geometric way to define the $\alpha$-invariant over an arbitrary field of characteristic zero.

Definition 1.2. Let $X$ be a Fano orbifold defined over $k$. The global log canonical threshold of $X$ is defined by the number

$$
\alpha(X)=\sup \left\{\begin{array}{l|l}
\lambda \in \mathbb{Q} & \begin{array}{l}
\text { the pair }(X, \lambda D) \text { is log canonical for every effective } \\
\mathbb{Q} \text {-divisor } D \text { numerically equivalent to }-K_{X} .
\end{array}
\end{array} .\right.
$$

It has been verified that for a Fano orbifold defined over $\mathbb{C}$ its $\alpha$-invariant coincides with the global log canonical threshold ( [5]). For this reason, the same name $\alpha$-invariant and the same notation $\alpha(X)$ will be used for the global log canonical threshold of the Fano orbifold defined over $k$ in the present article.

The original purpose of the $\alpha$-invariant is to show the existence of Kähler-Einstein metrics on given Fano manifolds ( [24]). Fujita, Odaka and Sano however reinterpret the $\alpha$-invariant as a sufficient condition for a $\mathbb{Q}$-Fano variety to be K-stable.

Theorem 1.3 ( [20], [11]). Let $X$ be a $\mathbb{Q}$-Fano variety. Either if

$$
\alpha(X)>\frac{\operatorname{dim}(X)}{\operatorname{dim}(X)+1}
$$

or if $\alpha(X)=\frac{\operatorname{dim}(X)}{\operatorname{dim}(X)+1}$ and $X$ is smooth, then the pair $\left(X,-K_{X}\right)$ is K-stable.

This enables us to have a detour studying K-stability for some specific $\mathbb{Q}$-Fano varieties. Even though the method is only a one-side implication and the $\alpha$-invariants are not easy to compute at all, this is the only pragmatic way, known to us so far, to verify K-stability in concrete cases. It is an indisputable expectation that computing the $\alpha$-invariant of a given Fano variety should be much more doable than directly investigating its degenerations.

For instance, Cheltsov has computed the exact values of the $\alpha$-invariants of all the smooth del Pezzo surfaces. For the purpose of K-stability, we summarize his computation in the following way.

Theorem 1.5 ( [1]). Let $S$ be a smooth del Pezzo surface of degree d.

- If $d \geq 5$, then $\alpha(S)<\frac{2}{3}$.

- If $d \leq 4$, then $\alpha(S) \geq \frac{2}{3}$.

With this estimation, the method of Fujita-Odaka-Sano immediately implies that a smooth del Pezzo surface of degree at most 4 is K-stable with respect to its anticanonical polarisation.

Even though we are not able to completely determine the K-stability of all the smooth del Pezzo surfaces with their $\alpha$-invariants, this result demonstrates that the $\alpha$-invariant is a very practical tool to test K-stability for given Fano varieties. The following higher dimensional smooth Fano varieties are also instructive examples to which we can apply the method of FujitaOdaka-Sano:

- a double cover of $\mathbb{P}^{n}$ ramified along a smooth hypersurface of degree $2 n, n \geq 3$;

- a smooth hypersurface of degree $n$ in $\mathbb{P}^{n}, n \geq 4$.

All the smooth Fano varieties in these families satisfy the condition of Theorem 1.3 ( [3], [2]). Therefore, they are K-stable, and hence the Fano manifolds defined over $\mathbb{C}$ in these families admit Kähler-Einstein metrics. In particular, a smooth hypersurface of degree $n$ in $\mathbb{P}^{n}$ with generalized Eckardt points ( [2]) is an example of a Kähler-Einstein Fano manifold whose Kähler-Einstein metric is verified to exist only by proving its K-stability ( [2], 4], [11]).

Recently Fujita and Odaka provided a new algebro-geometric way to test K-(semi)stability of Fano varieties. To introduce their method, let $X$ be a $\mathbb{Q}$-factorial variety with at worst $\log$ 
canonical singularities, $Z \subset X$ a closed subvariety and $D$ an effective $\mathbb{Q}$-divisor on $X$. The $\log$ canonical threshold of $D$ along $Z$ is the number

$$
c_{Z}(X, D)=\sup \{c \mid \text { the pair }(X, c D) \text { is } \log \text { canonical along } Z \text {. }\} \text {. }
$$

Because log canonicity is a local property, we see that

$$
c_{Z}(X, D)=\inf _{p \in Z}\left\{c_{p}(X, D)\right\}
$$

If $X=\mathbb{A}^{n}$ and $D=(f=0)$, where $f$ is a polynomial defined over $\mathbb{A}^{n}$, then we also use the notation $c_{0}(f)$ for the $\log$ canonical threshold of $D$ at the origin.

Definition 1.6 ( $[13])$. Let $X$ be a $\mathbb{Q}$-Fano variety and let $m$ be a positive integer such that the plurianticanonical linear system $\left|-m K_{X}\right|$ is non-empty. Set $\ell_{m}=h^{0}\left(X, \mathcal{O}_{X}\left(-m K_{X}\right)\right)$. For a section $s$ in $\mathrm{H}^{0}\left(X, \mathcal{O}_{X}\left(-m K_{X}\right)\right)$, we denote the effective divisor of the section $s$ by $D(s)$. If $\ell_{m}$ sections $s_{1}, \ldots, s_{\ell_{m}}$ form a basis of the space $\mathrm{H}^{0}\left(X, \mathcal{O}_{X}\left(-m K_{X}\right)\right)$, then the anticanonical $\mathbb{Q}$-divisor

$$
D:=\frac{1}{\ell_{m}} \sum_{i=1}^{\ell_{m}} \frac{1}{m} D\left(s_{i}\right)
$$

is said to be of $m$-basis type. For a positive integer $m$, we set

$$
\delta_{m}(X)=\inf _{\substack{D: \\ m \text {-basis type }}} c_{X}(X, D) .
$$

We set $\delta_{m}(X)=0$ if $\left|-m K_{X}\right|$ is empty. The $\delta$-invariant of $X$ is defined by the number

$$
\delta(X)=\limsup _{m} \delta_{m}(X) .
$$

Using the $\delta$-invariant, Fujita and Odaka set up a conjectural criterion for K-(semi)stability. They then proved that the $\delta$-invariant gives a sufficient condition for K-(semi)stability.

Conjecture 1.7 ( [13]). A $\mathbb{Q}$-Fano variety $X$ is K-stable (resp. K-semistable) with respect to $-K_{X}$ if and only if $\delta(X)>1$ (resp. $\geq 1$ ).

Note that Conjecture 1.7 is true if Berman-Gibbs stability ( [12]) is equivalent to K-stability.

Theorem 1.8 ( $[13)$. Let $X$ be a $\mathbb{Q}$-Fano variety. If $\delta(X)>1$ (resp. $\geq 1)$, then $\left(X,-K_{X}\right)$ is K-stable (resp. K-semistable).

Proof. See [13].

In the present article we utilize this theorem to verify the K-(semi)stability of smooth del Pezzo surfaces, i.e., we prove the following.

Main Theorem. Let $S$ be a smooth del Pezzo surface of degree d.

- If $d \leq 5$, then $\delta(S) \geq \frac{15}{14}>1$.

- If $S \cong \mathbb{P}^{2}, \mathbb{P}^{1} \times \mathbb{P}^{1}$ or the del Pezzo surface of degree 6 , then $\delta(S)=1$.

- If $S \cong \mathbb{F}_{1}$ or the del Pezzo surface of degree 7 , then $\delta(S)<1$.

Through Theorem 1.8, these estimations of the $\delta$-invariants immediately yield the following.

Corollary 1.9. Let $S$ be a smooth del Pezzo surface of degree $d$. Then the pair

$$
\left(S,-K_{S}\right) \text { is }\left\{\begin{array}{l}
K \text {-semistable if } S \cong \mathbb{P}^{2}, \mathbb{P}^{1} \times \mathbb{P}^{1} \text { or the del Pezzo surface of degree } 6 . \\
K \text {-stable if } d \leq 5 .
\end{array}\right.
$$

Since the sufficient condition for K-stability given by the $\alpha$-invariant in (1.4) involves the dimensions of Fano varieties, the following theorem shows that the $\alpha$-invariant itself is paralyzed in testing K-stability of products of Fano varieties. 
Theorem 1.10. Let $X$ and $Y$ be smooth Fano varieties. Then

$$
\alpha(X \times Y)=\min \{\alpha(X), \alpha(Y)\} .
$$

Proof. See [5, Lemma 2.29].

We strongly believe that the same formula holds for the $\delta$-invariant.

Conjecture 1.11. Let $X$ and $Y$ be smooth Fano varieties. Then

$$
\delta(X \times Y)=\min \{\delta(X), \delta(Y)\} .
$$

Remark 1.12. One can easily verify the conjecture in case when either $X$ or $Y$ is 1-dimensional, i.e., $\mathbb{P}^{1}$.

From this formula we see that the $\delta$-invariant, unlike the $\alpha$-invariant, keeps its ability to test K-stability in products of Fano varieties. For instance, Main Theorem implies the following.

Corollary 1.13. Assume that Conjecture 1.11 holds. Then a product of smooth del Pezzo surfaces of degrees $\leq 5$ is K-stable. A product of K-semistable smooth del Pezzo surfaces is K-semistable.

It is certain that the $\delta$-invariant produces a new method to verify existence of Kähler-Einstein metrics on Fano varieties through Theorem 1.1. It is no exaggeration to say that this new method is simpler and more coherent than the method by the $\alpha$-invariant.

Corollary 1.14. A smooth del Pezzo surface of degree $\leq 5$ defined over $\mathbb{C}$ admits a KählerEinstein metric.

Since Conjecture 1.7 has not been verified completely, at this moment we cannot say that the last statement of Main Theorem implies that the Hirzebruch surface $\mathbb{F}_{1}$ and the del Pezzo surface of degree 7 are not K-semistable. Yet it should be remarked here that algebro-geometric ways to prove their non-K-semistability are already known.

Proposition 1.15. The del Pezzo surfaces of degree 7 and the Hirzebruch surface $\mathbb{F}_{1}$ are not K-semistable.

Proof. For instance, see [10, Examples 6.5 and 6.5], [15, Proposition 3.1], [21]

Since the del Pezzo surfaces in the second statement of Main Theorem are all toric varieties, they cannot be K-stable. Therefore, Corollary 1.9 and Proposition 1.15 verify that Conjecture 1.7 holds good for 2-dimensional Fano manifolds.

\section{Preliminaries}

Let $f$ be a polynomial over the field $k$ in variables $z_{1}, \ldots, z_{n}$. Assign positive integral weights $w\left(z_{i}\right)$ to the variables $z_{i}$. Let $w(f)$ be the weighted multiplicity of $f$ at the origin (= the lowest weight of the monomials occurring in $f$ ) and let $f_{w}$ denote the weighted homogeneous leading term of $f$ ( $=$ the term of the monomials in $f$ with the weighted multiplicity of $f$ ).

Let $g$ be a polynomial over the field $k$ in $z_{2}, \ldots, z_{n}$ and set

$$
h\left(z_{1}, \ldots, z_{n}\right)=f\left(z_{1}+g\left(z_{2}, \ldots, z_{n}\right), z_{2}, \ldots, z_{n}\right) .
$$

If $z_{1}+g\left(z_{2}, \ldots, z_{n}\right)$ is weighted homogeneous with respect to the given weights $w\left(z_{1}\right), \ldots, w\left(z_{n}\right)$, it is clear that

$$
h_{w}\left(z_{1}, \ldots, z_{n}\right)=f_{w}\left(z_{1}+g\left(z_{2}, \ldots, z_{n}\right), z_{2}, \ldots, z_{n}\right) .
$$

Let $f_{1}, \ldots, f_{\ell}$ be polynomials over the field $k$ in $z_{1}, \ldots, z_{n}$. With respect to the given weights $w\left(z_{1}\right), \ldots, w\left(z_{n}\right)$, we easily see that

$$
\left(\prod_{i=1}^{\ell} f_{i}\right)_{w}=\prod_{i=1}^{\ell}\left(f_{i}\right)_{w}, \quad w\left(\prod_{i=1}^{\ell} f_{i}\right)=\sum_{i=1}^{\ell} w\left(f_{i}\right) .
$$


Let $\mathfrak{m} \subset k\left[z_{1}, \ldots, z_{n}\right]$ be the maximal ideal of the origin in $\mathbb{A}^{n}$. Let $f_{1}, \ldots, f_{\ell}$ be polynomials over the field $k$ in $z_{1}, \ldots, z_{n}$ that induce a basis for the $d$-jet space at the origin, i.e., $k\left[z_{1}, \ldots, z_{n}\right] / \mathfrak{m}^{d+1}$, where $d$ is a positive integer and $\ell=\operatorname{dim}_{k} k\left[z_{1}, \ldots, z_{n}\right] / \mathfrak{m}^{d+1}$. The $k$-linear map of $k\left[z_{1}, \ldots, z_{n}\right] / \mathfrak{m}^{d+1}$ induced by the coordinate change $z_{1}-g\left(z_{2}, \ldots, z_{n}\right) \mapsto z_{1}$ and $z_{i} \mapsto z_{i}$ for $i \geq 2$ is an automorphism. Therefore, the new polynomials $f_{1}\left(z_{1}+\right.$ $\left.g\left(z_{2}, \ldots, z_{n}\right), z_{2}, \ldots, z_{n}\right), \ldots, f_{\ell}\left(z_{1}+g\left(z_{2}, \ldots, z_{n}\right), z_{2}, \ldots, z_{n}\right)$ also induce a basis for the $d$-jet space.

Proposition 2.1. Let $f$ be a polynomial over $\mathbb{A}^{n}$. Assign integral weights $w\left(z_{i}\right)$ to the variables and let $w(f)$ be the weighted multiplicity of $f$. Then

- $c_{0}\left(f_{w}\right) \leq c_{0}(f) \leq \frac{\sum w\left(z_{i}\right)}{w(f)}$.

- If

$$
\left(\mathbb{A}^{n}, \frac{\sum w\left(z_{i}\right)}{w(f)} \cdot\left(f_{w}=0\right)\right)
$$

is log canonical outside the origin, then $c_{0}(f)=\frac{\sum w\left(z_{i}\right)}{w(f)}$.

Proof. See [17, Propositions 8.13 and 8.14].

\section{K-Semistable del Pezzo surfaces I}

Fix a positive integer $m$ and put $\ell_{m}=h^{0}\left(\mathbb{P}^{2}, \mathcal{O}_{\mathbb{P}^{2}}\left(-m K_{\mathbb{P}^{2}}\right)\right)$. Let $[x: y: z]$ be a homogeneous coordinate for $\mathbb{P}^{2}$.

Since the $\ell_{m}$ monomials of degree $3 m$ in $x, y, z$ form a basis of the space $\mathrm{H}^{0}\left(\mathbb{P}^{2}, \mathcal{O}_{\mathbb{P}^{2}}\left(-m K_{\mathbb{P}^{2}}\right)\right)$, the effective divisor defined by the equation $x y z=0$ is an anticanonical divisor of $m$-basis type. This shows that

$$
\delta_{m}\left(\mathbb{P}^{2}\right) \leq 1
$$

for each $m$. Therefore, $\delta\left(\mathbb{P}^{2}\right) \leq 1$.

Theorem 3.1. The $\delta$-invariant of $\mathbb{P}^{2}$ is 1 .

Proof. Let $\left\{s_{1}, \ldots, s_{\ell_{m}}\right\}$ be a basis of $\mathrm{H}^{0}\left(\mathbb{P}^{2}, \mathcal{O}_{\mathbb{P}^{2}}\left(-m K_{\mathbb{P}^{2}}\right)\right)$. We denote the effective divisor of the section $s_{i}$ by $D_{i}$ and set

$$
D:=\sum_{i=1}^{\ell_{m}} D_{i} .
$$

It is enough to show that for an arbitrary point $p$ in $\mathbb{P}^{2}$

$$
c_{p}\left(\mathbb{P}^{2}, D\right) \geq \frac{1}{m \ell_{m}} .
$$

By a linear coordinate change, we may assume that $p=[0: 0: 1]$.

Consider the $\ell_{m}$ monomials of degree $3 m$ in the variables $x, y, z$. Putting $z=1$, we obtain the $\ell_{m}$ monomials of degrees at most $3 m$

$$
1, x, y, x^{2}, x y, x y^{2}, \ldots, x^{k}, x^{k-1} y, \ldots, x y^{k-1}, y^{k}, \ldots, x^{3 m}, x^{3 m-1} y, \ldots, x y^{3 m-1}, y^{3 m},
$$

where they are written in lexicographic order. In this order, denote the $i$-th monomial by $\mathbf{x}_{i}$ for each $i=1, \ldots, \ell_{m}$.

We may consider $x$ and $y$ as local coordinates around the point $p$. Then each $D_{i}$ is defined around the point $p$ by a polynomial $f_{i}$ of degree at most $3 m$ in the variables $x, y$. The divisor $D$ is defined by $f:=\prod f_{i}$ in an affine neighborhood of $p$.

Since the sections $s_{1}, \ldots, s_{\ell_{m}}$ form a basis for $\mathrm{H}^{0}\left(\mathbb{P}^{2}, \mathcal{O}_{\mathbb{P}^{2}}\left(-m K_{\mathbb{P}^{2}}\right)\right)$ and they induce the polynomials $f_{1}, \ldots, f_{\ell_{m}}$, respectively, we may assume that $f_{i}$ contains the monomial $\mathbf{x}_{i}$ for each $i=1, \ldots, \ell_{m}$. 
Now we consider the Newton polygon of the polynomial $f$ in $\mathbb{R}^{2}$, where we use coordinate functions $(s, t)$ for $\mathbb{R}^{2}$.

Claim 1. The Newton polygon of the polynomial $f$ contains the point $\left(m \ell_{m}, m \ell_{m}\right)$ corresponding to the monomial $x^{m \ell_{m}} y^{m \ell_{m}}$.

Since each $f_{i}$ contains the monomial $\mathbf{x}_{i}$, we have $w\left(f_{i}\right) \leq w\left(\mathbf{x}_{i}\right)$ with respect to given weights $w(x), w(y)$, and hence $w(f) \leq w\left(\prod \mathbf{x}_{i}\right)=w\left(x^{m \ell_{m}} y^{m \ell_{m}}\right)$. This proves the claim.

Claim 2. If the line $s=t$ intersects the Newton polygon of $f$ at one of its vertices, then $c_{0}(f) \geq \frac{1}{m \ell_{m}}$.

In such a case, we can take weights $w(x), w(y)$ such that $f_{w}$ is of the form $\epsilon x^{a} y^{a}$, where $\epsilon$ is a non-zero constant. Claim 1 implies that $a \leq m \ell_{m}$. We then obtain

$$
c_{0}(f) \geq c_{0}\left(f_{w}\right)=\frac{1}{a} \geq \frac{1}{m \ell_{m}}
$$

from Proposition 2.1,

We may therefore assume that any vertex of the Newton polygon of $f$ does not lie on the line $s=t$.

Step A. Let $\Lambda$ be the edge of the Newton polygon of $f$ that intersects the line $s=t$. Let $w(x), w(y)$ be the weights such that the monomials of $f_{w}$ are plotted on the edge $\Lambda$.

If $\Lambda$ is either vertical ( $\left.f_{w}=x^{a} g(y)\right)$ or horizontal $\left(f_{w}=y^{a} h(x)\right)$, where $g$ and $h$ are polynomials of multiplicity at most $a-1$ at the origin, then Claim 1 implies $a \leq m \ell_{m}$. It then follows from Proposition 2.1 that $c_{0}(f) \geq \frac{1}{m \ell_{m}}$.

Therefore, we may assume that $\Lambda$ is neither vertical nor horizontal. The slope of the edge $\Lambda$ is equal to $-\frac{w(x)}{w(y)}$.

Step B. We write an irreducible decomposition of $f_{w}$ as

$$
f_{w}=\epsilon x^{a} y^{b}\left(x^{\alpha_{1}}+g_{1}(x, y)\right)^{c_{1}} \cdots\left(x^{\alpha_{r}}+g_{r}(x, y)\right)^{c_{r}},
$$

where $\epsilon$ is a non-zero constant and $g_{i}(x, y)$ is an irreducible weighted homogeneous polynomial of degree $w\left(x^{\alpha_{i}}\right)$ such that it does not contain the monomial $x^{\alpha_{i}}$. Note that $a, b \leq m \ell_{m}$.

Let $c=\max \left\{c_{i}\right\}$. We may assume that $c_{1}=c$. For the convenience, we set $\alpha=\alpha_{1}$. Since $g_{1}(x, y)$ is irreducible, it must possess the monomial $y^{\beta}$ for some positive integer $\beta$.

Claim 3. If $c \leq m \ell_{m}$, then $c_{0}(f) \geq \frac{1}{m \ell_{m}}$.

It immediately follows from Proposition 2.1 that

$$
c_{0}\left(f_{w}\right)=\min \left\{\frac{1}{a}, \frac{1}{b}, \frac{1}{c}, \frac{w(x)+w(y)}{w\left(f_{w}\right)}\right\} .
$$

By Claim 1, $\frac{w(x)+w(y)}{w\left(f_{w}\right)} \geq \frac{1}{m \ell}$, so that $c_{0}\left(f_{w}\right) \geq \frac{1}{m \ell}$. Therefore, $c_{0}(f) \geq c_{0}\left(f_{w}\right) \geq \frac{1}{m \ell}$.

Step C. Suppose that $c>m \ell_{m}$. Since $w\left(\left(x^{\alpha}+y^{\beta}\right)^{c}\right) \leq w\left(x^{m \ell_{m}} y^{m \ell_{m}}\right)$ by Claim 1, if $\alpha, \beta \geq 2$, then we immediately obtain $c \leq m \ell_{m}$. Therefore, either $\alpha=1$ or $\beta=1$. By exchanging coordinates if necessary, we may assume that $\alpha=1$. Note that $\alpha=1$ implies that $w(x) \geq w(y)$ and $\frac{w(x)}{w(y)}$ is the integral number $\beta$.

Therefore,

$$
f_{w}=\epsilon x^{a} y^{b}\left(x+A_{1} y^{\beta}\right)^{c}\left(x^{\alpha_{2}}+g_{2}(x, y)\right)^{c_{2}} \cdots\left(x^{\alpha_{r}}+g_{r}(x, y)\right)^{c_{r}},
$$


where $A_{1}$ is a non-zero constant. The weighted leading term $f_{w}$ contains the monomial $x^{\left(a+c+\sum_{i=2}^{r} \alpha_{i} c_{i}\right)} y^{b}$ and $a+c+\sum_{i=2}^{r} \alpha_{i} c_{i} \geq c>m \ell_{m}$.

We now apply a change of coordinate $x+A_{1} y^{\beta} \mapsto x$ to the polynomials $f_{i}$ and $f$. Set

$$
f_{i}^{(1)}(x, y):=f_{i}\left(x-A_{1} y^{\beta}, y\right)
$$

for each $i$ and

$$
f^{(1)}(x, y):=f\left(x-A_{1} y^{\beta}, y\right) .
$$

Then $f_{w}^{(1)}(x, y)=f_{w}\left(x-A_{1} y^{\beta}, y\right)$ and $f^{(1)}=\prod f_{i}^{(1)}$.

Since $f_{1}^{(1)}, \ldots, f_{\ell_{m}}^{(1)}$ form a basis for the $(3 m)$-jet space $k[x, y] /(x, y)^{3 m+1}$, we may again assume that $f_{i}^{(1)}$ contains the monomial $\mathbf{x}_{i}$ for each $i$. The Newton polygon of the polynomial $f^{(1)}$ again contains the point corresponding to $x^{m \ell_{m}} y^{m \ell_{m}}$.

Now we go back to Step A with $f^{(1)}$ instead of $f$, i.e., let $\Lambda^{(1)}$ be the edge of the Newton polygon of $f^{(1)}$ that intersects the line $s=t$. We also find weights $w^{(1)}(x), w^{(1)}(y)$ with respect to which the monomials of $f_{w^{(1)}}^{(1)}$ are plotted on the edge $\Lambda^{(1)}$. The slope of the edge $\Lambda^{(1)}$ is $-\frac{w^{(1)}(x)}{w^{(1)}(y)}$.

Let $L$ be the line in $\mathbb{R}^{2}$ determined by the edge $\Lambda$. We observe that

there is no monomial in $f^{(1)}$ plotted under the line $L$ and that there is no monomial in $f^{(1)}$ plotted on the line $L$ with $s<c$.

But $f^{(1)}$ possesses the monomial $x^{a+c+\sum_{i=2}^{r} \alpha_{i} c_{i}} y^{b}$ that is plotted on $L$. This shows that $\Lambda^{(1)}$ is strictly steeper than $\Lambda$, i.e., $-\frac{w^{(1)}(x)}{w^{(1)}(y)}<-\frac{w(x)}{w(y)}$.

As before, we write

$$
f_{w^{(1)}}^{(1)}=\epsilon^{(1)} x^{a^{(1)}} y^{b^{(1)}}\left(x^{\alpha_{1}^{(1)}}+g_{1}^{(1)}(x, y)\right)^{c_{1}^{(1)}} \cdots\left(x_{r^{(1)}}^{\alpha^{(1)}}+g_{r^{(1)}}^{(1)}(x, y)\right)^{c_{r(1)}^{(1)}},
$$

where $\epsilon^{(1)}$ is a non-zero constant and $g_{i}^{(1)}(x, y)$ is an irreducible weighted homogeneous polynomial of degree $w^{(1)}\left(x^{\alpha_{i}^{(1)}}\right)$ such that it does not contain the monomial $x^{\alpha_{i}^{(1)}}$.

Let $c^{(1)}=\max \left\{c_{i}^{(1)}\right\}$. Again we assume that $c^{(1)}=c_{1}^{(1)}$. The irreducible polynomial $g_{1}^{(1)}$ must contain the monomial $y^{\beta^{(1)}}$ for some positive integer $\beta^{(1)}$.

If $c^{(1)} \leq m \ell_{m}$, then the proof is done by Claim 3. If $c^{(1)}>m \ell_{m}$, then we follow Step C. We here remark that $\alpha^{(1)}$ must be 1 because $\frac{w^{(1)}(x)}{w^{(1)}(y)}>\frac{w(x)}{w(y)} \geq 1$. Note $\frac{w^{(1)}(x)}{w^{(1)}(y)}$ is the integral number $\beta^{(1)}$ and $\frac{w^{(1)}(x)}{w^{(1)}(y)}-\frac{w(x)}{w(y)}=\beta^{(1)}-\beta \geq 1$. Now we go back to Step A with the new coordinate-changed polynomials $f_{i}^{(2)}$ and $f^{(2)}$.

For the proof, it is enough to show that this procedure terminates in a finite number of loops. The slope of $\Lambda^{(i)}$ is bounded below by $-m \ell_{m}$ since the Newton polygon of $f^{(i)}$ must contain the point corresponding to $x^{m \ell_{m}} y^{m \ell_{m}}$ and it has the property $(*)$ above. The termination is therefore guaranteed because the slope of $\Lambda^{(i)}$ drops by at least 1 for each loop.

\section{K-stable del Pezzo surfaces}

In this section, we prove the first statement of Main Theorem. Before we start, we should remark here that the estimations of the $\delta$-invariants in this section are not sharp at all. Since we have only to check that the $\delta$-invariants are strictly bigger than 1 , our estimations have been made in such a way that we can reduce the amount of computation as much as possible.

Let $S_{d}$ be a smooth del Pezzo surface of degree $d \leq 5$. Let $m$ be a positive integer and let $s_{1}, \ldots, s_{\ell_{m}}$ be sections in $\mathrm{H}^{0}\left(S_{d}, \mathcal{O}_{S_{d}}\left(-m K_{S_{d}}\right)\right)$ that form a basis for $\mathrm{H}^{0}\left(S_{d}, \mathcal{O}_{S_{d}}\left(-m K_{S_{d}}\right)\right)$, 
where $\ell_{m}=\frac{d m(m+1)}{2}+1$. Denote the divisor of the section $s_{i}$ by $D_{i}^{m}$. Set

$$
D^{m}:=\sum_{i=1}^{\ell_{m}} D_{i}^{m}
$$

Since $d \leq 5$, for a given point $p \in S_{d}$, we can choose mutually disjoint $(9-d)(-1)$-curves $M_{1}, \ldots, M_{9-d}$ which do not pass through the point $p$. By contracting these $(-1)$-curves, we obtain a birational morphism $\pi: S_{d} \rightarrow \mathbb{P}^{2}$ such that it is an isomorphism in a neighborhood of $p$. Set $q=\pi(p)$. By a suitable coordinate change, we may assume that $q=[0: 0: 1]$. Note that $c_{p}\left(S_{d}, D_{i}^{m}\right)=c_{q}\left(\mathbb{P}^{2}, \pi\left(D_{i}^{m}\right)\right)$ for $i=1, \ldots, \ell_{m}$ and $c_{p}\left(S_{d}, D^{m}\right)=c_{q}\left(\mathbb{P}^{2}, \pi\left(D^{m}\right)\right)$. Denote $\pi\left(D_{i}^{m}\right)$ by $\bar{D}_{i}^{m}$ for each $i$ and $\pi\left(D^{m}\right)$ by $\bar{D}^{m}$.

For an effective divisor $C$ in $\left|-m K_{S_{d}}\right|, \pi(C)$ is an effective divisor of degree $3 m$ on $\mathbb{P}^{2}$ which passes through the points $\pi\left(M_{1}\right), \ldots, \pi\left(M_{9-d}\right)$ with multiplicities at least $m$. Such divisors produce an $\ell_{m}$-dimensional subspace of $\mathrm{H}^{0}\left(\mathbb{P}^{2}, \mathcal{O}_{\mathbb{P}^{2}}\left(-m K_{\mathbb{P}^{2}}\right)\right)$. We denote this subspace by $\mathcal{L}_{m}$. The effective divisors $\bar{D}_{1}^{m}, \ldots, \bar{D}_{\ell_{m}}^{m}$ induce a basis for $\mathcal{L}_{m}$.

As before, let $[x: y: z]$ be a homogeneous coordinate for $\mathbb{P}^{2}$. We may consider $x$ and $y$ as local coordinates in the affine chart $U$ by $z \neq 0$. For a polynomial $g(x, y)=g_{i}(x, y)+$ $g_{i+1}(x, y)+\ldots$, where $g_{j}(x, y)$ is a homogeneous polynomial of degree $j$, we will call the lowest degree term $g_{i}(x, y)$ the Zariski tangent term of $g(x, y)$. In $U$, the divisor $\bar{D}_{i}^{m}$ is defined by a polynomial $f_{m, i}$. Note that

$$
m \ell_{m} \cdot c_{0}\left(\prod_{i=1}^{\ell_{m}} f_{m, i}\right)=c_{q}\left(\mathbb{P}^{2}, \frac{1}{m \ell_{m}} \bar{D}^{m}\right)=c_{p}\left(S_{d}, \frac{1}{m \ell_{m}} D^{m}\right) .
$$

For each $0 \leq n \leq 3 m$, we define a subspace of the $k$-vector space of homogeneous polynomials of degree $n$ in the variables $x$ and $y$ as follows:

$$
\mathcal{T}_{n, m}:=\left\{\begin{array}{l|l}
g(x, y) \in k[x, y]_{n} & \begin{array}{l}
\text { there is an effective divisor } C \text { in }\left|-m K_{S_{d}}\right| \text { such that } \\
\text { the divisor } \pi(C) \text { is defined in } U \text { by a polynomial } \\
\text { whose Zariski tangent term is } g(x, y) .
\end{array}
\end{array}\right\},
$$

where $k[x, y]_{n}$ denotes the space of homogeneous polynomials of degree $n$ in $x, y$. Note that the space $k[x, y]_{n}$ is spanned by the standard basis

$$
\mathcal{S}_{n}=\left\{x^{n}, x^{n-1} y, \ldots, x y^{n-1}, y^{n}\right\} .
$$

Set

$$
\mathcal{T}_{m}:=\bigoplus_{n=0}^{3 m} \mathcal{T}_{n, m}
$$

A basis for $\mathcal{T}_{m}$ consisting only of homogeneous elements will be called a basis for $\mathcal{T}_{m}$.

Put $\ell=\operatorname{dim}_{k}\left(\mathcal{T}_{m}\right)$. Let $\left\{t_{1}, \ldots, t_{\ell}\right\}$ be a basis of $\mathcal{T}_{m}$. For each $1 \leq j \leq \ell$ we take a polynomial $h_{m, j}$ on $U$ originated from $\mathcal{L}_{m}$ such that its Zariski tangent term is $t_{j}$. It is obvious that $h_{m, 1}, \ldots, h_{m, \ell}$ are linearly independent, so that $\ell \leq \ell_{m}$. Let $G$ be a member in $\mathcal{L}_{m}$. It can be expressed on $U$ by a polynomial $g(x, y)=g_{i}(x, y)+g_{i+1}(x, y)+\ldots$, where $g_{j}(x, y)$ is a homogeneous polynomial of degree $j$. If $i=3 m$, then $g(x, y)$ is a linear combination of $h_{m, 1}, \ldots, h_{m, \ell}$. Assume that if $a+1 \leq i \leq 3 m$, then $g(x, y)$ is a linear combination of $h_{m, 1}, \ldots, h_{m, \ell}$. For $i=a$, we have constants $\alpha_{1}, \ldots, \alpha_{\ell}$ such that the Zariski tangent term of $\sum \alpha_{j} h_{j}(x, y)$ is $g_{i}(x, y)$. Since $g(x, y)-\sum \alpha_{j} h_{m, j}(x, y)$ is induced by some element in $\mathcal{L}_{m}$, the polynomial $g(x, y)$ is a linear combination of $h_{m, 1}, \ldots, h_{m, \ell}$. Thus inductively we can conclude that every polynomial on $U$ from $\mathcal{L}_{m}$ is a linear combination of $h_{m, 1}, \ldots, h_{m, \ell}$. Consequently, the dimension of $\mathcal{T}_{m}$ is equal to the dimension of $\mathrm{H}^{0}\left(S_{d}, \mathcal{O}_{S_{d}}\left(-m K_{S_{d}}\right)\right)$, i.e., $\ell=\ell_{m}$. 
Now let us explain how to estimate the log canonical threshold

$$
c_{0}\left(\prod_{i=1}^{\ell_{m}} f_{m, i}\right) \text {. }
$$

Step 0. Consider the space $\mathcal{L}_{1}$. Using a suitable coordinate change, we choose a basis $\left\{t_{1}, \ldots, t_{d+1}\right\}$ for the space $\mathcal{T}_{1}$ with $\operatorname{deg}\left(t_{1}\right) \leq \ldots \leq \operatorname{deg}\left(t_{d+1}\right)$ such that $t_{1}, \ldots, t_{d}$ are monomials. Indeed, in each proof, we will immediately see that this is possible whenever we need this step. Denote the monomial $t_{i}$ by $\mathbf{x}_{1, i}$ for $i=1, \ldots, d$. Choose an appropriate monomial from those in $t_{d+1}$ and denote it by $\mathbf{x}_{1, d+1}$.

Step 1. Consider the finite sets

$$
\begin{aligned}
& \mathcal{C}_{m}:=\left\{\prod_{i=1}^{d+1} \mathbf{x}_{1, i}^{n_{i}} \mid n_{i} \text { are non-negative integers with } n_{1}+\cdots+n_{d+1}=m\right\}, \\
& \mathcal{B}_{m}^{s}:=\left\{\prod_{i=1}^{d+1} t_{i}^{n_{i}} \mid n_{i} \text { are non-negative integers with } n_{1}+\cdots+n_{d+1}=m\right\} .
\end{aligned}
$$

The monomial $\prod_{i=1}^{d+1} \mathbf{x}_{1, i}^{n_{i}}$ is contained in the homogeneous polynomial $\prod_{i=1}^{d+1} t_{i}^{n_{i}}$. Since the sets $\left\{t_{1}, \ldots, t_{d+1}\right\}$ and $\left\{\mathbf{x}_{1,1}, \ldots, \mathbf{x}_{1, d+1}\right\}$ are linearly independent respectively, the sets $\mathcal{C}_{m}$ and $\mathcal{B}_{m}^{s}$ are linearly independent respectively.

Denote the number of elements in $\mathcal{B}_{m}^{s}$ by $b$. Note that $b=\left(\begin{array}{c}m+d \\ d\end{array}\right) \leq \ell_{m}$. Set $a=\ell_{m}-b$.

Step 2. We choose linearly independent $a$ homogeneous elements $u_{1}, \ldots, u_{a}$ in $\mathcal{T}_{m}$ such that the set $\left\{u_{1}, \ldots, u_{a}\right\} \cup \mathcal{B}_{m}^{s}$ forms a basis for $\mathcal{T}_{m}$.

Lemma 4.1. There is an injective map

$$
\iota_{m}:\left\{u_{1}, \ldots, u_{a}\right\} \cup \mathcal{B}_{m}^{s} \rightarrow \bigcup_{n=0}^{3 m} \mathcal{S}_{n}
$$

such that

(1) $\iota_{m}\left(\prod_{i=1}^{d+1} t_{i}^{n_{i}}\right)=\prod_{i=1}^{d+1} \mathbf{x}_{1, i}^{n_{i}}$;

(2) $\iota_{m}\left(u_{i}\right)$ is contained in the homogeneous polynomial $u_{i}$ for each $i$.

Proof. See Appendix at the end.

Step 3. Consider all the possibilities of the image $\iota_{m}\left(\left\{u_{1}, \ldots, u_{a}\right\}\right)$ with linearly independent $a$ homogeneous elements $u_{1}, \ldots, u_{a}$ in $\mathcal{T}_{m}$ such that the set $\left\{u_{1}, \ldots, u_{a}\right\} \cup \mathcal{B}_{m}^{s}$ forms a basis for $\mathcal{T}_{m}$.

Lemma 4.3. There is an injective map

$$
I_{m}:\left\{f_{m, i} \mid 1 \leq i \leq \ell_{m}\right\} \rightarrow \bigcup_{n=0}^{3 m} \mathcal{S}_{n}
$$

such that

(1) the monomial $I_{m}\left(f_{m, i}\right)$ is contained in $f_{m, i}$;

(2) its image coincides with the image of $\iota_{m}$.

Proof. See Appendix at the end. 
Lemma 4.3 implies that the Newton polygon of the polynomial $\prod_{i=1}^{\ell_{m}} f_{m, i}$ contains the point corresponding to the monomial

$$
x^{\alpha} y^{\beta}:=\prod_{i=1}^{\ell_{m}} I_{m}\left(f_{m, i}\right)=\prod_{i=1}^{a} \iota_{m}\left(u_{i}\right) \cdot \prod_{\mathbf{x} \in \mathcal{C}_{m}} \mathbf{x} .
$$

Varying the possible image $\iota_{m}\left(\left\{u_{1}, \ldots, u_{a}\right\}\right)$, we find an attainable maximum $v_{m}$ of the value $\max \{\alpha, \beta\}$. Then we see that the Newton polygon of $\prod_{i=1}^{\ell_{m}} f_{m, i}$ always contains the point corresponding to the monomial $x^{v_{m}} y^{v_{m}}$.

Step 4. With the monomial $x^{v_{m}} y^{v_{m}}$, we can follow Steps A, B, C in the proof of Theorem 3.1 . Then we obtain

This implies

$$
c_{0}\left(\prod_{i=1}^{\ell_{m}} f_{m, i}\right) \geq \frac{1}{v_{m}}
$$

$$
m \ell_{m} \cdot c_{0}\left(\prod_{i=1}^{\ell_{m}} f_{m, i}\right)=c_{q}\left(\mathbb{P}^{2}, \frac{1}{m \ell_{m}} \bar{D}^{m}\right)=c_{p}\left(S_{d}, \frac{1}{m \ell_{m}} D^{m}\right) \geq \frac{m \ell_{m}}{v_{m}}
$$

for an arbitrary point $p$ on $S_{d}$. Therefore,

$$
\delta\left(S_{d}\right) \geq \limsup _{m} \frac{m \ell_{m}}{v_{m}} .
$$

In Step $\mathrm{C}$ we define the polynomials $f_{i}^{(1)}$ by applying a change of coordinate $x+A_{1} y^{\beta} \mapsto x$ to the polynomial $f_{i}$ for each $i$. We furthermore define $f_{i}^{(k)}$ inductively for each $i$ and each $k$. We here apply the same to $f_{m, i}$ and denote the coordinate-changed polynomials by $f_{m, i}^{(k)}$. In the change of coordinate $x+A_{1} y^{\beta} \mapsto x$, we may assume that $\beta>1$. Indeed, if $\beta=1$, then we replace $f_{m, i}(x, y)$ by $f_{m, i}\left(x-A_{1} y, x\right)$. Then they also form a basis for $\mathcal{L}_{m}$ and the log canonical threshold of their product at the origin is equal to that of the product of the original $f_{m, i}$. Note that the edge of the Newton polygon of the product of the replaced $f_{m, i}$ that intersects the line $s=t$ is not parallel to the line $s=-t$. This implies that $\beta$ for the replaced $f_{m, i}$ cannot be 1 in Step C. Therefore, it is strictly bigger than 1 and hence $\beta^{(k)}$ for $f_{m}^{(k)}:=\prod_{i=1}^{\ell_{m}} f_{m, i}^{(k)}$ is also strictly bigger than 1 for all $k$. The lemma below guarantees that we are able to go through Step $\mathrm{C}$ in Step 4.

Lemma 4.6. For each $k$ there is an injective map

$$
I_{m, k}:\left\{f_{m, i}^{(k)} \mid 1 \leq i \leq \ell_{m}\right\} \rightarrow \bigcup_{n=0}^{3 m} \mathcal{S}_{n}
$$

such that

(1) the monomial $I_{m, k}\left(f_{m, i}^{(k)}\right)$ is contained in $f_{m, i}^{(k)}$;

(2) its image coincides with the image of $\iota_{m}$.

Proof. See Appendix at the end.

Note that in some cases, we may skip Steps 0 and 1, i.e., we may start from Step 2 with $a=\ell_{m}$.

As we see, the $\delta$-invariant is defined in an asymptotic way. Therefore, it is enough to see the asymptotic behaviors of $m \ell_{m}$ and $v_{m}$ with respect to $m$. Since $m \ell_{m}=\frac{d}{2} m^{3}+\frac{d}{2} m^{2}+m$, a lower bound of the $\delta$-invariant of $S_{d}$ can be determined by the coefficient of the term $\mathrm{m}^{3}$ in $v_{m}$. For reader's convenience, we here provide the following diagrams. In each diagram, the 
exponent of $x$ in the product of all the monomials in $x, y$ corresponding to the integral points of a triangle (or a quadrilateral) is given by a cubic polynomial with respect to $m$. The number in the triangle (or the quadrilateral) is the coefficient of the term $\mathrm{m}^{3}$ in this cubic polynomial. The diagrams in Figure 1 enable us to immediately figure out the coefficients of the term $\mathrm{m}^{3}$ in the exponents $v_{m}$ that appear in the present and the next sections.

FiguRE 1.
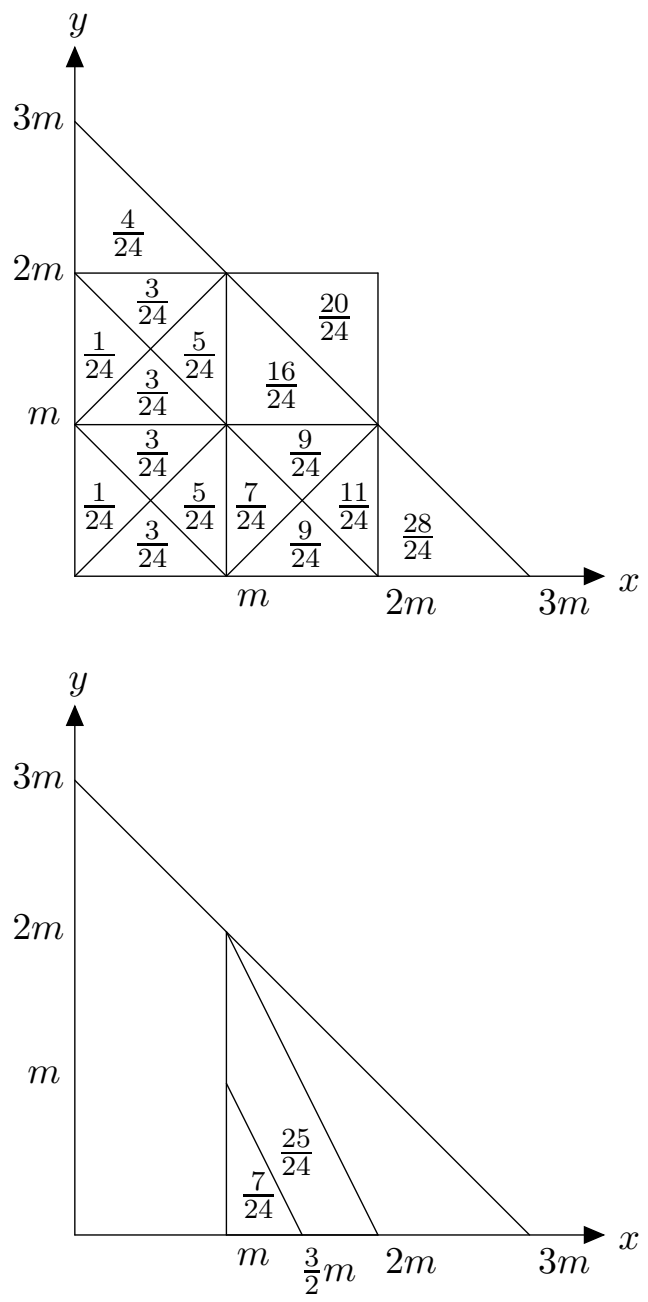
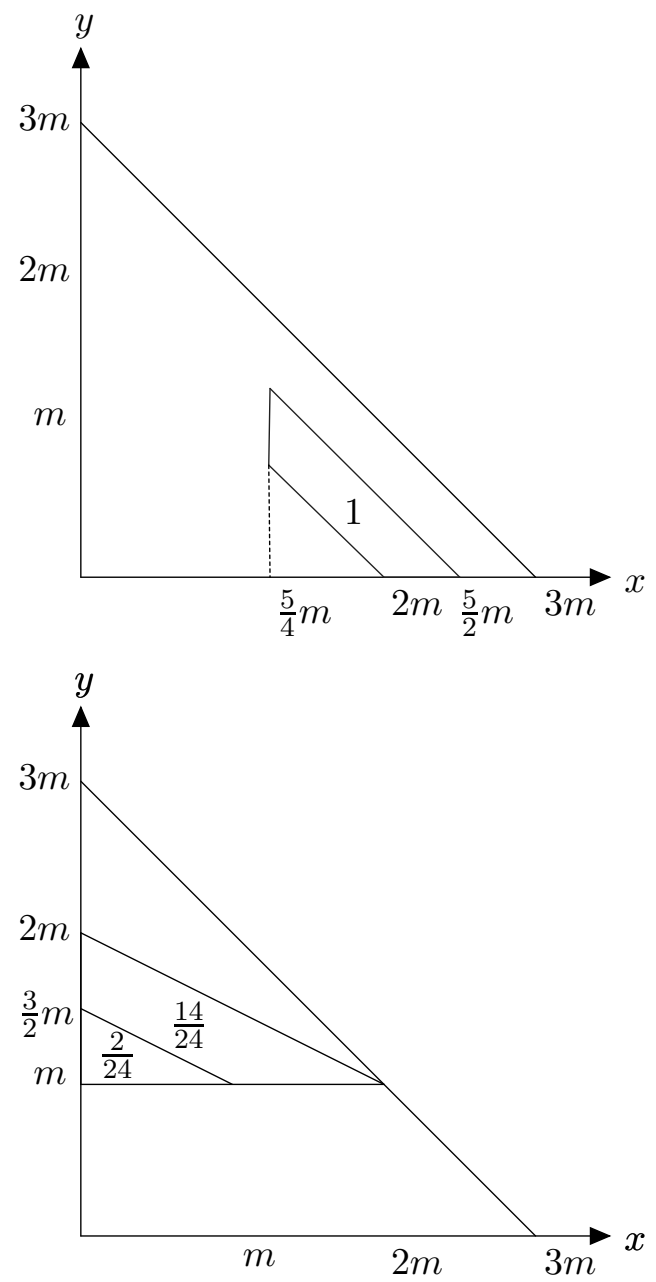

Now we are ready to estimate the values of the $\delta$-invariants of smooth del Pezzo surfaces. We keep the same notations as before.

Theorem 4.7. Let $S$ be a del Pezzo surface of degree 1 . Then $\delta(S) \geq \frac{3}{2}$.

Proof. Let $C$ be a cubic curve in $\mathbb{P}^{2}$ that passes through the points $\pi\left(M_{1}\right), \ldots, \pi\left(M_{8}\right)$ and $q$. Since $\pi\left(M_{1}\right), \ldots, \pi\left(M_{8}\right)$ are in general position, the curve $C$ must be irreducible and reduced.

Case 1. The curve $C$ is smooth at $q$.

The effective divisor $G$ produced by a section in $\mathcal{L}_{m}$ can pass through the point $q$ with multiplicity at most $m$. To see this, we write $G=m^{\prime} C+\Omega$, where $m^{\prime}$ is a non-negative 
integer not bigger than $m$ and $\Omega$ is an effective divisor whose support does not contain $C$. If $\operatorname{mult}_{q}(G)>m$, then $\operatorname{mult}_{q}(\Omega)>m-m^{\prime}$. This yields an absurd inequality

$$
9\left(m-m^{\prime}\right)=C \cdot \Omega \geq \operatorname{mult}_{q}(\Omega)+\sum_{i=1}^{8} \operatorname{mult}_{\pi\left(M_{i}\right)}(C) \cdot \operatorname{mult}_{\pi\left(M_{i}\right)}(\Omega)>9\left(m-m^{\prime}\right) .
$$

Therefore, the image of the injective map in (4.2) is always contained in the set

$$
\left\{x^{n_{1}} y^{n_{2}} \mid 0 \leq n_{1}+n_{2} \leq m\right\} .
$$

These monomials are plotted in the shade area of the following diagram:

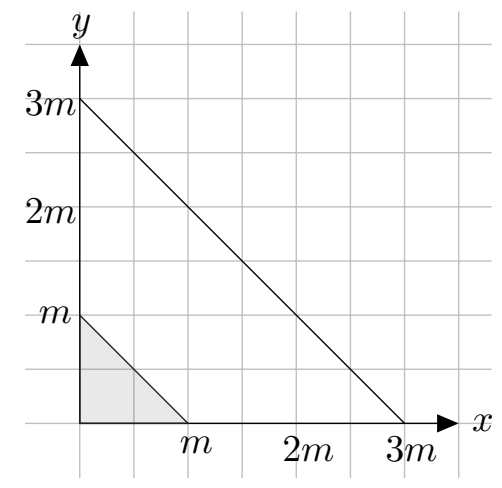

Since $m \ell_{m}=\frac{1}{2} m\left(m^{2}+m+2\right)$, Figure 1 shows

$$
\limsup _{m} \frac{m \ell_{m}}{v_{m}}=3
$$

Case 2. The curve $C$ is singular at $q$.

Then $C$ is a unique curve in $\mathbb{P}^{2}$ that passes through the points $\pi\left(M_{1}\right), \ldots, \pi\left(M_{8}\right)$ and $q$. Note that $\operatorname{dim}_{k} \mathcal{T}_{1}=2$. Using a suitable coordinate change, if $C$ has a node at $q$, then we may assume that

$$
t_{1}=1, t_{2}=x y,
$$

and if $C$ has a cusp at $q$, then we may assume that

$$
t_{1}=1, t_{2}=(x+y)^{2} .
$$

Therefore, in both the cases we can always take

$$
\mathbf{x}_{1,1}=1, \mathbf{x}_{1,2}=x y .
$$

We then obtain

$$
\mathcal{C}_{m}=\left\{1, x y, x^{2} y^{2}, \ldots, x^{m} y^{m}\right\} .
$$

Let $G$ be the effective divisor of a section in $\mathcal{L}_{m}$. We write $G=m^{\prime} C+\Omega$ as in Case 1 . Since $9\left(m-m^{\prime}\right)=C \cdot \Omega \geq 8\left(m-m^{\prime}\right)+2 \operatorname{mult}_{q}(\Omega)$, we obtain

$$
\operatorname{mult}_{q}(G)=m^{\prime} \cdot \operatorname{mult}_{q}(C)+\operatorname{mult}_{q}(\Omega) \leq 2 m^{\prime}+\frac{m-m^{\prime}}{2} .
$$

This shows that we can define the injective map $\iota_{m}$ in (4.2) in such a way that its image is contained in the set

$$
\bigcup_{i=0}^{m}\left\{x^{i+n_{1}} y^{i+n_{2}} \mid 0 \leq n_{1}, n_{2} \text { and } 0 \leq n_{1}+n_{2} \leq \frac{m-i}{2}\right\} .
$$

These monomials are plotted in the shade area of the following diagram: 


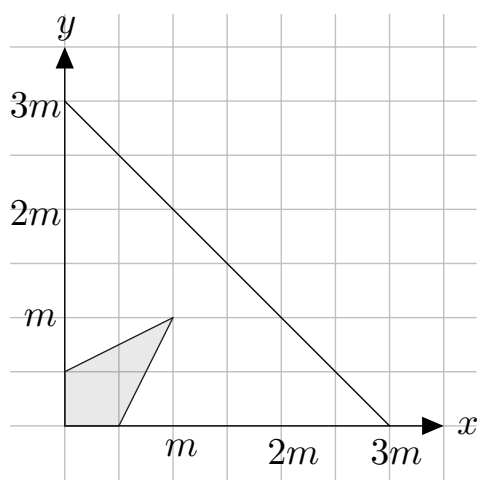

The exponent of $x$ in the product of all the monomials corresponding to the integral points of the shade area in the diagram above is clearly smaller than the exponent from the shade area in the below.

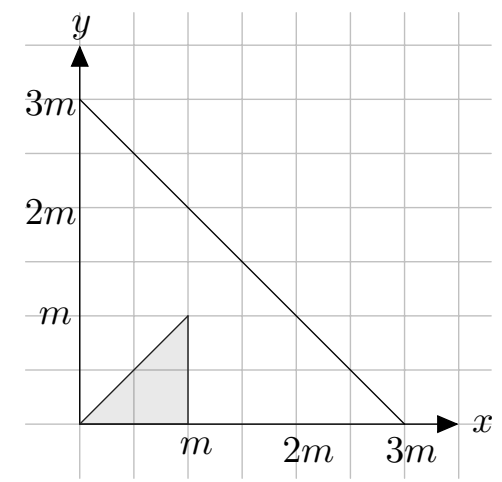

Therefore, Figure 1 shows

$$
\limsup _{m} \frac{m \ell_{m}}{v_{m}} \geq \frac{3}{2}
$$

Consequently, from Case 1 and Case 2 we obtain $\delta(S) \geq \frac{3}{2}$.

Theorem 4.8. Let $S$ be a del Pezzo surface of degree 2. Then $\delta(S) \geq \frac{6}{5}$.

Proof. Note that $\operatorname{dim}_{k}\left(\mathcal{T}_{1}\right)=3$. We have two possibilities: either $\operatorname{deg}\left(t_{1}\right)=0, \operatorname{deg}\left(t_{2}\right)=$ $\operatorname{deg}\left(t_{3}\right)=1$ or $\operatorname{deg}\left(t_{1}\right)=0, \operatorname{deg}\left(t_{2}\right)=1, \operatorname{deg}\left(t_{3}\right)=2$.

Case 1. $\operatorname{deg}\left(t_{1}\right)=0, \operatorname{deg}\left(t_{2}\right)=\operatorname{deg}\left(t_{3}\right)=1$.

In this case, we may assume that

$$
t_{1}=1, t_{2}=x, t_{3}=y
$$

by a suitable coordinate change. Therefore, $\mathbf{x}_{1,1}=1, \mathbf{x}_{1,2}=x$ and $\mathbf{x}_{1,3}=y$. We then obtain

$$
\mathcal{C}_{m}=\left\{1, x, y, x^{2}, x y, y^{2}, \ldots, x^{m}, x^{m-1} y, \ldots, y^{m}\right\} .
$$

We claim that the effective divisor $G$ yielded by a section in $\mathcal{L}_{m}$ cannot pass through $q$ with multiplicity more than $2 m$. For the claim, we consider the effective plurianticanonical divisor

$$
\tilde{G}=\pi^{*}(G)-m \sum_{i=1}^{7} M_{i}
$$

on $S$. Since $2 m=H \cdot \tilde{G} \geq \operatorname{mult}_{p}(\tilde{G})$ for a general member $H$ in $\left|-K_{S}\right|$ passing through the point $p$, we obtain $\operatorname{mult}_{p}(\tilde{\tilde{G}}) \leq 2 m$. This implies the claim. 

in

Therefore, the image of $\left\{u_{1}, \ldots, u_{a}\right\}$ under the injective map $\iota_{m}$ in (4.2) is always contained

$$
\bigcup_{n=0}^{2 m} \mathcal{S}_{n} \backslash \mathcal{C}_{m}
$$

where $a=\ell_{m}-\frac{(m+1)(m+2)}{2}=\frac{m(m-1)}{2}$. In case when

$$
\iota_{m}\left(\left\{u_{1}, \ldots, u_{a}\right\}\right)=\left\{x^{n_{1}} y^{n_{2}} \mid 0 \leq n_{1}, n_{2}, m+2 \leq n_{1}+n_{2} \leq 2 m \text { and } m+2 \leq n_{1} \leq 2 m\right\}
$$

the value $\alpha$ in the monomial $x^{\alpha} y^{\beta}$ of (4.5) attains the possible maximum $v_{m}$. Therefore, the value $v_{m}$ can be asymptotically evaluated by the shade area in the following diagram:

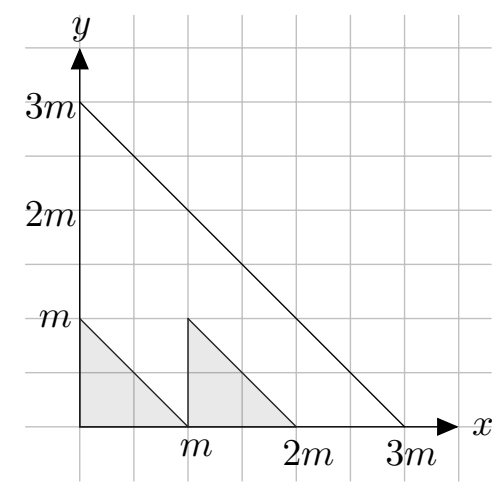

i.e., Figure 1 shows

$$
\limsup _{m} \frac{m \ell_{m}}{v_{m}}=\frac{6}{5}
$$

Case 2. $\operatorname{deg}\left(t_{1}\right)=0, \operatorname{deg}\left(t_{2}\right)=1, \operatorname{deg}\left(t_{3}\right)=2$.

In this case, there is a unique cubic $C$ on $\mathbb{P}^{2}$ that passes through the points $\pi\left(M_{1}\right), \ldots, \pi\left(M_{7}\right)$ and that has multiplicity 2 at the point $q$.

Let $G$ be the effective divisor of a section in $\mathcal{L}_{m}$.

Subcase 1. The curve $C$ is irreducible.

In this subcase, we may assume that the Zariski tangent term of the defining polynomial of $C$ on $U$ contains the monomial $x y$.

We write $G=m^{\prime} C+\Omega$, where $m^{\prime}$ is a non-negative integer not bigger than $m$ and $\Omega$ is an effective divisor whose support does not contain $C$. Since

$$
9\left(m-m^{\prime}\right)=C \cdot \Omega \geq 7\left(m-m^{\prime}\right)+2 \operatorname{mult}_{q}(\Omega),
$$

the injective map $\iota_{m}$ in (4.2) can be defined in a way that its image is contained in the set

$$
\bigcup_{i=0}^{m}\left\{x^{i+n_{1}} y^{i+n_{2}} \mid 0 \leq n_{1}, n_{2} \text { and } 0 \leq n_{1}+n_{2} \leq m-i\right\} .
$$

This set can be depicted as follows: 


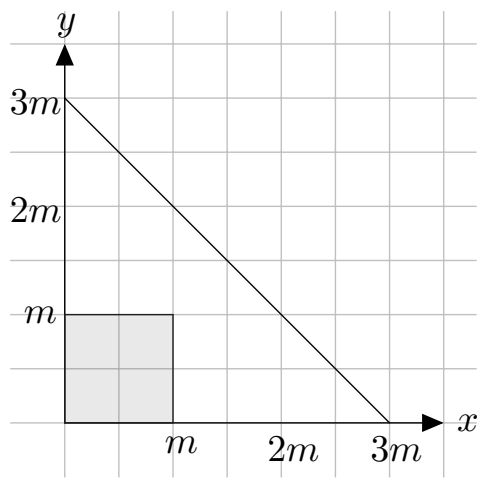

Figure 1 shows

$$
\limsup _{m} \frac{m \ell_{m}}{v_{m}}=2
$$

Subcase 2. The curve $C$ is reducible.

The cubic $C$ consists of a line $L$ and an irreducible conic $Q$. Since $\left(-K_{S}\right)^{2}=2$, it cannot consist of three lines. Note that $L$ passes through exactly two points of $\pi\left(M_{1}\right), \ldots, \pi\left(M_{7}\right)$ and that $Q$ passes through five of them. We may assume that the Zariski tangent term of the defining polynomial of $L$ on $U$ contains the monomial $x$ and that of $Q$ contains the monomial $y$.

We write $G=m_{1} L+m_{2} Q+\Omega$, where $m_{1}$ and $m_{2}$ are non-negative integers and $\Omega$ is an effective divisor whose support contains neither $L$ nor $Q$.

From the inequalities

$$
\begin{aligned}
& 3 m=L \cdot\left(m_{1} L+m_{2} Q+\Omega\right) \geq m_{1}+2 m_{2}+2\left(m-m_{1}\right)+\text { mult }_{q} \Omega, \\
& 6 m=Q \cdot\left(m_{1} L+m_{2} Q+\Omega\right) \geq 2 m_{1}+4 m_{2}+5\left(m-m_{2}\right)+\text { mult }_{q} \Omega,
\end{aligned}
$$

we obtain $-m_{1}+2 m_{2} \leq m$ and $2 m_{1}-m_{2} \leq m$. These imply $m_{1}, m_{2} \leq m$. Moreover, we obtain

$$
\operatorname{mult}_{q}(\Omega) \leq m+\min \left\{-2 m_{1}+m_{2}, m_{1}-2 m_{2}\right\} \text {. }
$$

Therefore, we can define the injective map $\iota_{m}$ of (4.2) in a way that its image is contained in the sets either

or

$$
\bigcup_{0 \leq m_{1} \leq m_{2} \leq m}\left\{x^{m_{1}+n_{1}} y^{m_{2}+n_{2}} \mid 0 \leq n_{1}, n_{2} \text { and } 0 \leq n_{1}+n_{2} \leq m+m_{1}-2 m_{2}\right\}
$$

$$
\bigcup_{0 \leq m_{2} \leq m_{1} \leq m}\left\{x^{m_{1}+n_{1}} y^{m_{2}+n_{2}} \mid 0 \leq n_{1}, n_{2} \text { and } 0 \leq n_{1}+n_{2} \leq m-2 m_{1}+m_{2}\right\} .
$$

Both the sets sit in the shade area of the following diagram:

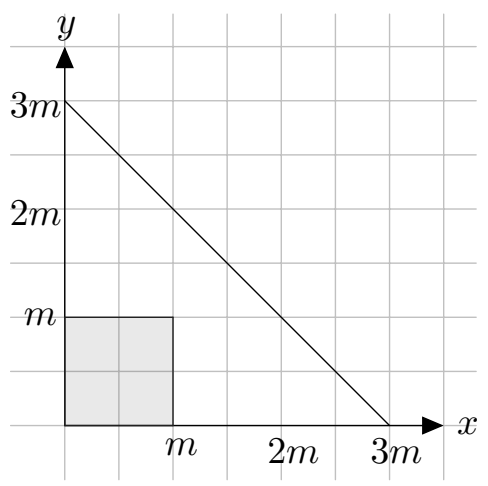


Therefore,

$$
\limsup _{m} \frac{m \ell_{m}}{v_{m}}=2
$$

Consequently, from Cases 1 and 2 we obtain $\delta(S) \geq \frac{6}{5}$.

Theorem 4.9. Let $S$ be a del Pezzo surface of degree 3 . Then $\delta(S) \geq \frac{36}{31}$.

Proof. Let $C$ be the unique cubic on $\mathbb{P}^{2}$ that passes through the points $\pi\left(M_{1}\right), \ldots, \pi\left(M_{6}\right)$ and that is singular at the point $q$. Let $G$ be the effective divisor of a section in $\mathcal{L}_{m}$.

Case 1. The curve $C$ is irreducible.

In this case, the Zariski tangent term of the defining polynomial of $C$ on $U$ may be assumed to contain the monomial $x y$.

We write $G=m^{\prime} C+\Omega$, where $m^{\prime}$ is a non-negative integer not bigger than $m$ and $\Omega$ is an effective divisor whose support does not contain $C$. Since

$$
9\left(m-m^{\prime}\right)=C \cdot \Omega \geq 6\left(m-m^{\prime}\right)+2 \operatorname{mult}_{q}(\Omega),
$$

we have

$$
\operatorname{mult}_{q}(\Omega) \leq \frac{3}{2}\left(m-m^{\prime}\right)
$$

This implies that the injective map $\iota_{m}$ in (4.2) can be chosen in such a way that its image is contained in the set

$$
\bigcup_{i=0}^{m}\left\{x^{i+n_{1}} y^{i+n_{2}} \mid 0 \leq n_{1}, n_{2} \text { and } 0 \leq n_{1}+n_{2} \leq \frac{3}{2}(m-i)\right\} .
$$

These monomials are plotted in the shade area as below:

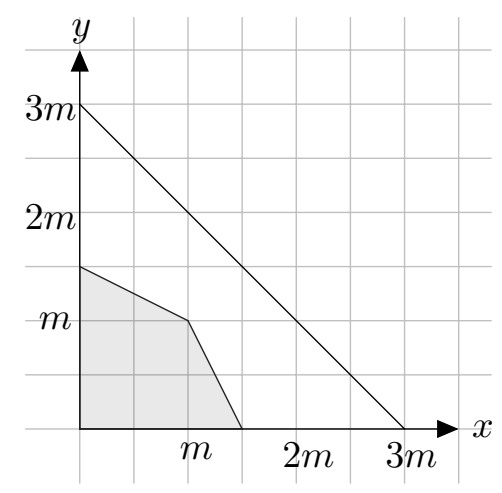

We then obtain

$$
\limsup _{m} \frac{m \ell_{m}}{v_{m}}=\frac{12}{7}
$$

from Figure 1 .

Case 2. The curve $C$ is reducible.

The cubic curve $C$ then consists of either one line and one irreducible conic or three lines.

Subcase 1. The curve $C$ consists of a line $L$ and an irreducible conic $Q$.

In this subcase, we may assume that the Zariski tangent term of the defining polynomial of $L$ on $U$ contains the monomial $x$ and that of $Q$ contains the monomial $y$.

We write $G=m_{1} L+m_{2} Q+\Omega$, where $m_{1}$ and $m_{2}$ are non-negative integers and $\Omega$ is an effective divisor whose support contains neither $L$ nor $Q$. 
We first suppose that $L$ passes through exactly one of the points $\pi\left(M_{1}\right), \ldots, \pi\left(M_{6}\right)$. From

$$
\begin{gathered}
6 m=Q \cdot\left(m_{1} L+m_{2} Q+\Omega\right) \geq 2 m_{1}+4 m_{2}+5\left(m-m_{2}\right)+\text { mult }_{q} \Omega, \\
3 m=L \cdot\left(m_{1} L+m_{2} Q+\Omega\right) \geq m_{1}+2 m_{2}+\left(m-m_{1}\right)+\text { mult }_{q} \Omega,
\end{gathered}
$$

we obtain $m_{2} \leq m, 2 m_{1}-m_{2} \leq m$ and

$$
\operatorname{mult}_{q} \Omega \leq \min \left\{m-2 m_{1}+m_{2}, 2 m-2 m_{2}\right\} .
$$

Therefore, the injective map $\iota_{m}$ in (4.2) can be defined in such a way that its image sits in the set

$$
\bigcup_{\substack{0 \leq m_{2} \leq m \\
2 m_{1}-m_{2} \leq m}}\left\{x^{m_{1}+n_{1}} y^{m_{2}+n_{2}} \mid 0 \leq n_{1}, n_{2} \text { and } 0 \leq n_{1}+n_{2} \leq \min \left\{\begin{array}{l}
m-2 m_{1}+m_{2} \\
2 m-2 m_{2}
\end{array}\right\}\right\} .
$$

It is easy to check that the monomials in this set are plotted in the shade area of the following diagram:

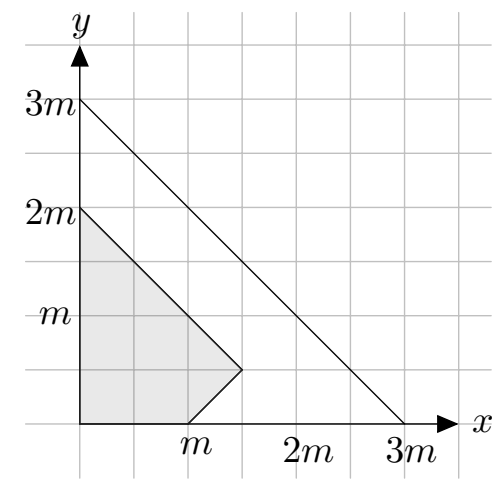

We now suppose that $L$ passes through two points of $\pi\left(M_{1}\right), \ldots, \pi\left(M_{6}\right)$. Then, from the inequalities

$$
\begin{aligned}
& 6 m=Q \cdot\left(m_{1} L+m_{2} Q+\Omega\right) \geq 2 m_{1}+4 m_{2}+4\left(m-m_{2}\right)+\text { mult }_{q} \Omega, \\
& 3 m=L \cdot\left(m_{1} L+m_{2} Q+\Omega\right) \geq m_{1}+2 m_{2}+2\left(m-m_{1}\right)+\text { mult }_{q} \Omega,
\end{aligned}
$$

we obtain $m_{1} \leq m, 2 m_{2}-m_{1} \leq m$ and

$$
\operatorname{mult}_{q} \Omega \leq \min \left\{m-2 m_{2}+m_{1}, 2 m-2 m_{1}\right\} .
$$

As the previous case, the injective map $\iota_{m}$ in (4.2) can be chosen in a way that its image is contained in the set

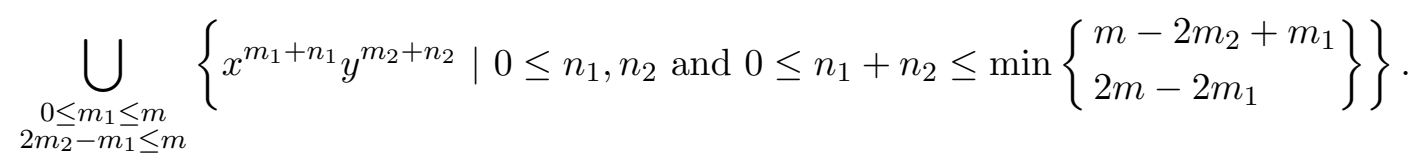

The monomials in this set are plotted in the shade area of the following diagram: 


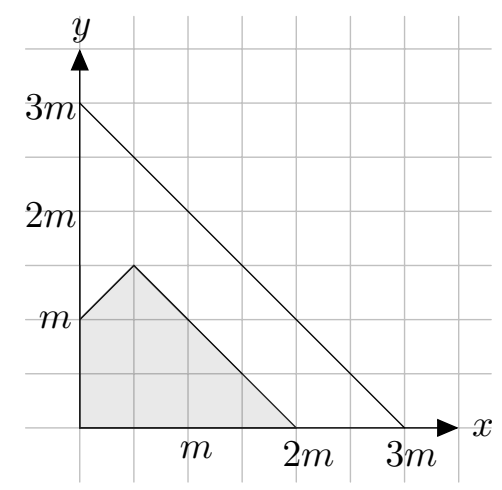

Therefore, Figure 1 implies

$$
\limsup _{m} \frac{m \ell_{m}}{v_{m}}=\frac{36}{31} .
$$

Subcase 2. The curve $C$ consists of three lines $L_{1}, L_{2}, L_{3}$ with $\operatorname{mult}_{q}(C)=2$.

We may assume that $q$ is the intersection point of $L_{1}$ and $L_{2}$. In addition, we may assume that $L_{1}$ is defined by $x=0$ and $L_{2}$ by $y=0$.

We write $G=m_{1} L_{1}+m_{2} L_{2}+m_{3} L_{3}+\Omega$, where $m_{1}, m_{2}, m_{3}$ are non-negative integers and $\Omega$ is an effective divisor whose support contains none of $L_{1}, L_{2}, L_{3}$. From the inequalities

$$
\begin{aligned}
3 m= & L_{1} \cdot\left(m_{1} L_{1}+m_{2} L_{2}+m_{3} L_{3}+\Omega\right) \geq m_{1}+m_{2}+m_{3}+2\left(m-m_{1}\right)+\operatorname{mult}_{q}(\Omega), \\
3 m= & L_{2} \cdot\left(m_{1} L_{1}+m_{2} L_{2}+m_{3} L_{3}+\Omega\right) \geq m_{1}+m_{2}+m_{3}+2\left(m-m_{2}\right)+\operatorname{mult}_{q}(\Omega), \\
& 3 m=L_{3} \cdot\left(m_{1} L_{1}+m_{2} L_{2}+m_{3} L_{3}+\Omega\right) \geq m_{1}+m_{2}+m_{3}+2\left(m-m_{3}\right),
\end{aligned}
$$

we see that the injective map $\iota_{m}$ in (4.2) can be defined in such a way that its image is contained in the set

$$
\bigcup_{\substack{0 \leq m_{1}, m_{2}, m_{3} \leq m \\
m_{1}+m_{2} \leq m_{3}+m}}\left\{x^{m_{1}+n_{1}} y^{m_{2}+n_{2}} \mid 0 \leq n_{1}, n_{2} \text { and } 0 \leq n_{1}+n_{2} \leq \min \left\{\begin{array}{l}
m-m_{1}+m_{2}-m_{3} \\
m+m_{1}-m_{2}-m_{3}
\end{array}\right\}\right\} .
$$

The monomials in this set are plotted in the shade area of the following diagram:

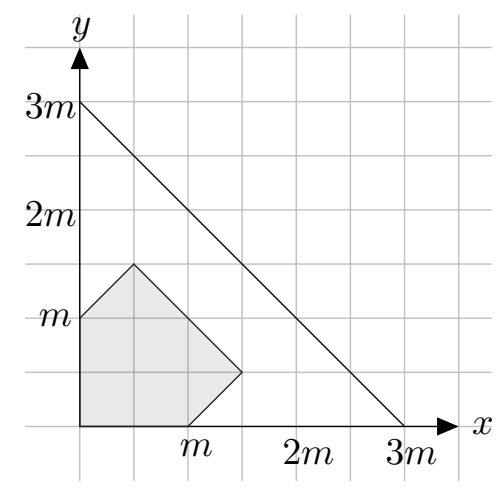

Therefore,

$$
\limsup _{m} \frac{m \ell_{m}}{v_{m}}=\frac{18}{11} .
$$

Subcase 3. The curve $C$ consists of three lines $L_{1}, L_{2}, L_{3}$ with $\operatorname{mult}_{q}(C)=3$.

The line $L_{1}$ can be assumed to be defined by $x=0, L_{2}$ by $y=0$ and $L_{3}$ by $x+y=0$. 
We write $G=m_{1} L_{1}+m_{2} L_{2}+m_{3} L_{3}+\Omega$, where $m_{1}, m_{2}, m_{3}$ are non-negative integers and $\Omega$ is an effective divisor whose support contains none of $L_{1}, L_{2}, L_{3}$. The three inequalities

$$
\begin{aligned}
& 3 m=L_{1} \cdot\left(m_{1} L_{1}+m_{2} L_{2}+m_{3} L_{3}+\Omega\right) \geq m_{1}+m_{2}+m_{3}+2\left(m-m_{1}\right)+\operatorname{mult}_{q}(\Omega), \\
& 3 m=L_{2} \cdot\left(m_{1} L_{1}+m_{2} L_{2}+m_{3} L_{3}+\Omega\right) \geq m_{1}+m_{2}+m_{3}+2\left(m-m_{2}\right)+\operatorname{mult}_{q}(\Omega), \\
& 3 m=L_{3} \cdot\left(m_{1} L_{1}+m_{2} L_{2}+m_{3} L_{3}+\Omega\right) \geq m_{1}+m_{2}+m_{3}+2\left(m-m_{3}\right)+\operatorname{mult}_{q}(\Omega)
\end{aligned}
$$

show that the injective map $\iota_{m}$ in (4.2) can be arranged in such a way that its image is contained in the set

$$
\bigcup_{0 \leq m_{1}, m_{2}, m_{3} \leq m}\left\{x^{m_{1}+m_{3}+n_{1}} y^{m_{2}+n_{2}} \mid 0 \leq n_{1}, n_{2} \text { and } 0 \leq n_{1}+n_{2} \leq \min \left\{\begin{array}{l}
m+m_{1}-m_{2}-m_{3} \\
m-m_{1}+m_{2}-m_{3} \\
m-m_{1}-m_{2}+m_{3}
\end{array}\right\}\right\} .
$$

The monomials in this set sit in the shade area in the diagram below:

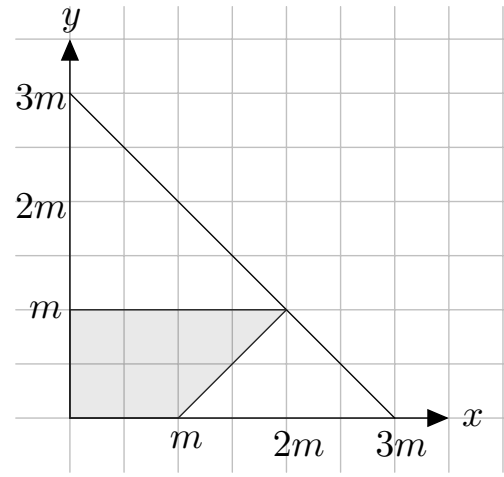

Figure 1 then implies

$$
\limsup _{m} \frac{m \ell_{m}}{v_{m}}=\frac{9}{7}
$$

Consequently, Cases 1 and 2 imply $\delta(S) \geq \frac{36}{31}$.

Theorem 4.10. Let $S$ be a del Pezzo surface of degree 4. Then $\delta(S) \geq \frac{12}{11}$.

Proof. Note that at most two (-1)-curves can pass through a given point $p$ on $S$.

Let $G$ be the effective divisor of a section in $\mathcal{L}_{m}$.

Case 1. There is no (-1)-curve that passes through the point $p$.

In this case, there is an irreducible cubic curve $C$ on $\mathbb{P}^{2}$ that passes through the points $\pi\left(M_{1}\right), \ldots, \pi\left(M_{5}\right)$ and that is singular at the point $q$. We may assume that the Zariski tangent term of the defining polynomial of $C$ on $U$ contains the monomial $x y$.

We write $G=m^{\prime} C+\Omega$, where $m^{\prime}$ is a non-negative integer not bigger than $m$ and $\Omega$ is an effective divisor whose support does not contain $C$. From the inequality

$$
9\left(m-m^{\prime}\right)=C \cdot \Omega \geq 5\left(m-m^{\prime}\right)+2 \operatorname{mult}_{q}(\Omega),
$$

we obtain

$$
\operatorname{mult}_{q}(\Omega) \leq 2\left(m-m^{\prime}\right)
$$

Therefore, the injective map $\iota_{m}$ in (4.2) can be chosen in such a way that its image is contained in the set

$$
\bigcup_{i=0}^{m}\left\{x^{i+n_{1}} y^{i+n_{2}} \mid 0 \leq n_{1}, n_{2} \text { and } 0 \leq n_{1}+n_{2} \leq 2(m-i)\right\} .
$$

The monomials in this set correspond to the integral points in the shade area below: 


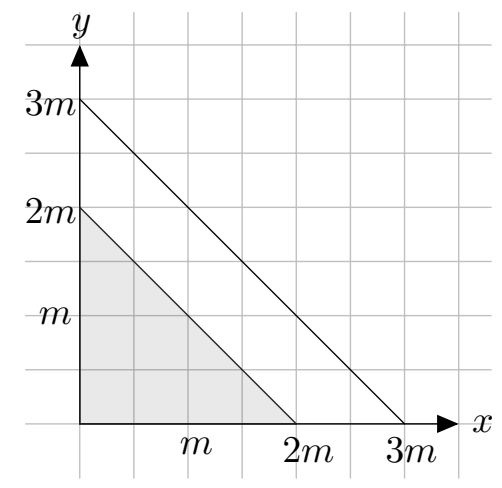

Therefore, Figure 1 implies

$$
\limsup _{m} \frac{m \ell_{m}}{v_{m}}=\frac{3}{2}
$$

Case 2. There is only one (-1)-curve that passes through the point $p$.

In this case, there is either a line passing through the point $q$ and two of the points $\pi\left(M_{1}\right), \ldots, \pi\left(M_{5}\right)$ or an irreducible conic passing through $q$ and $\pi\left(M_{1}\right), \ldots, \pi\left(M_{5}\right)$. We consider the former case only since the latter can be dealt in almost the same way. Let $L$ be the line in the former case. We may assume that it passes through $\pi\left(M_{1}\right)$ and $\pi\left(M_{2}\right)$. Also, there is an irreducible conic $Q$ that passes through the points $q$ and $\pi\left(M_{2}\right), \ldots, \pi\left(M_{5}\right)$. We write $G=m_{1} L+m_{2} Q+\Omega$, where $m_{1}, m_{2}$ are non-negative integers and $\Omega$ is an effective divisor whose support contains neither $L$ nor $Q$.

From the inequalities

$$
\begin{aligned}
& 3 m=L \cdot\left(m_{1} L+m_{2} Q+\Omega\right) \geq m_{1}+2 m_{2}+2\left(m-m_{1}\right)+\operatorname{mult}_{q}(\Omega), \\
& 6 m=Q \cdot\left(m_{1} L+m_{2} Q+\Omega\right) \geq 2 m_{1}+4 m_{2}+4\left(m-m_{2}\right)+\operatorname{mult}_{q}(\Omega),
\end{aligned}
$$

we obtain

$$
\operatorname{mult}_{q}(\Omega) \leq \min \left\{m+m_{1}-2 m_{2}, 2 m-2 m_{1}\right\}
$$

This implies that

$$
\operatorname{mult}_{q}(G)=m_{1}+m_{2}+\operatorname{mult}_{q}(\Omega) \leq \min \left\{m+2 m_{1}-m_{2}, 2 m-m_{1}+m_{2}\right\} \leq 2 m .
$$

Therefore, the image of the injective map $\iota_{m}$ in (4.2) is always contained in the same set as in the previous case, and hence

$$
\limsup _{m} \frac{m \ell_{m}}{v_{m}}=\frac{3}{2}
$$

Case 3. There are two (-1)-curves that pass through the point $p$.

There are two distinct lines $L_{1}, L_{2}$ passing through the point $q$ and two of the points $\pi\left(M_{1}\right), \ldots, \pi\left(M_{5}\right)$. We may assume that $L_{1}$ passes through $\pi\left(M_{1}\right), \pi\left(M_{2}\right)$ and that $L_{2}$ passes through $\pi\left(M_{3}\right), \pi\left(M_{4}\right)$. Let $L_{3}$ be the line determined by $q$ and $\pi\left(M_{5}\right)$.

By suitable coordinate changes, we may assume that the line $L_{1}$ is defined by $x=0, L_{2}$ by $y=0$ and $L_{3}$ by $x+y=0$. We then see that

$$
t_{1}=1, t_{2}=x, t_{3}=y, t_{4}=x y, t_{5}=x y(x+y)
$$

form a basis for $\mathcal{T}_{1}$. Furthermore, we may take

$$
\mathbf{x}_{1,1}=1, \mathbf{x}_{1,2}=x, \mathbf{x}_{1,3}=y, \mathbf{x}_{1,4}=x y, \mathbf{x}_{1,5}=x^{2} y .
$$


Then the set

$$
\mathcal{C}_{m}=\left\{\prod_{i=1}^{5} \mathbf{x}_{1, i}^{n_{i}} \mid n_{i} \text { are non-negative integers with } n_{1}+\cdots+n_{5}=m\right\}
$$

consists of the monomials corresponding the integral points in the shade area of the following diagram:

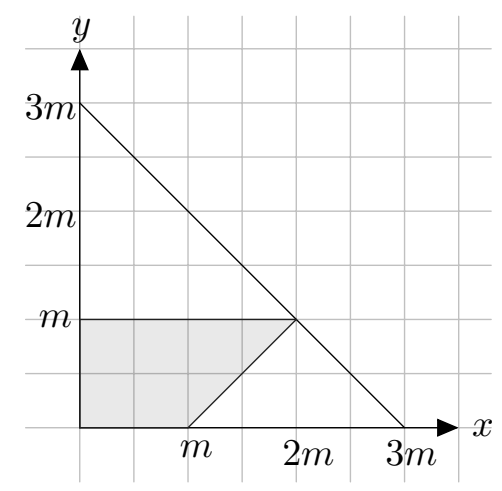

We now write $G=m_{1} L_{1}+m_{2} L_{2}+m_{3} L_{3}+\Omega$, where $m_{1}, m_{2}, m_{3}$ are non-negative integers and $\Omega$ is an effective divisor whose support contains none of $L_{1}, L_{2}, L_{3}$. The inequities

$$
\begin{gathered}
3 m=L_{1} \cdot\left(m_{1} L_{1}+m_{2} L_{2}+m_{3} L_{3}+\Omega\right) \geq m_{1}+m_{2}+m_{3}+2\left(m-m_{1}\right)+\operatorname{mult}_{q}(\Omega), \\
3 m=L_{2} \cdot\left(m_{1} L_{1}+m_{2} L_{2}+m_{3} L_{3}+\Omega\right) \geq m_{1}+m_{2}+m_{3}+2\left(m-m_{2}\right)+\operatorname{mult}_{q}(\Omega), \\
3 m=L_{3} \cdot\left(m_{1} L_{1}+m_{2} L_{2}+m_{3} L_{3}+\Omega\right) \geq m_{1}+m_{2}+m_{3}+\left(m-m_{3}\right)+\operatorname{mult}_{q}(\Omega)
\end{gathered}
$$

imply

$$
\operatorname{mult}_{q}(\Omega) \leq \min \left\{m+m_{1}-m_{2}-m_{3}, m-m_{1}+m_{2}-m_{3}, 2 m-m_{1}-m_{2}\right\} .
$$

This shows that the images $\iota_{m}\left(u_{1}\right), \ldots, \iota_{m}\left(u_{a}\right)$ can be plotted only above or on the line joining $(m, 0)$ and $(2 m, m)$. Since $\operatorname{deg}(G)=3 m$, they must sit only below or on the line joining $(3 m, 0)$ and $(0,3 m)$. Consequently, the maximum value $v_{m}$ can be attained when the image of the injective map $\iota_{m}$ in (4.2) are contained in the set of the monomials corresponding the integral points in the shade area of the following diagram:

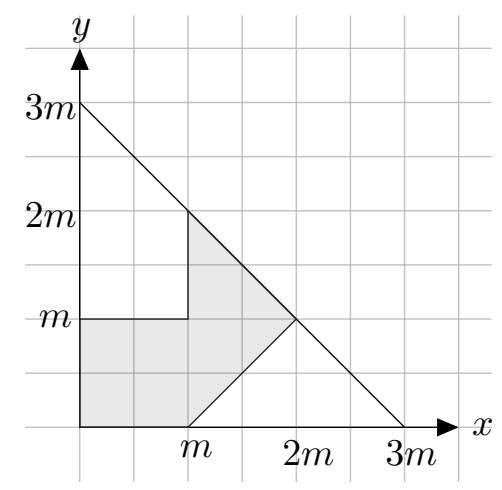

Therefore,

$$
\limsup _{m} \frac{m \ell_{m}}{v_{m}}=\frac{12}{11}
$$

Consequently, Cases 1,2 and 3 imply $\delta(S) \geq \frac{12}{11}$.

Theorem 4.11. Let $S$ be a del Pezzo surface of degree 5. Then $\delta(S) \geq \frac{15}{14}$. 
Proof. For a point $p$ on $S$, we may or may not have a member in $\left|-K_{S}\right|$ that has multiplicity 3 at $p$.

Case 1. No divisor in $\left|-K_{S}\right|$ has multiplicity 3 at $p$.

In this case, the monomials

$$
t_{1}=1, t_{2}=x, t_{3}=y, t_{4}=x^{2}, t_{5}=x y, t_{6}=y^{2}
$$

form a basis for $\mathcal{T}_{1}$. Therefore,

$$
\mathbf{x}_{1,1}=1, \mathbf{x}_{1,2}=x, \mathbf{x}_{1,3}=y, \mathbf{x}_{1,4}=x^{2}, \mathbf{x}_{1,5}=x y, \mathbf{x}_{1,6}=y^{2} .
$$

Then we obtain the set

$$
\mathcal{C}_{m}=\left\{x^{n_{1}} y^{n_{2}} \mid 0 \leq n_{1}, n_{2} \text { and } 0 \leq n_{1}+n_{2} \leq 2 m\right\} .
$$

Let $G$ be the effective divisor of a section in $\mathcal{L}_{m}$ and let $H$ be a general curve of degree 3 on $\mathbb{P}^{2}$ that has multiplicity 2 at $q$ and passes through $\pi\left(M_{1}\right), \pi\left(M_{2}\right), \pi\left(M_{3}\right)$ and $\pi\left(M_{4}\right)$. Then the inequality

$$
9 m \geq \operatorname{mult}_{q}(H) \operatorname{mult}_{q}(G)+\sum_{i=1}^{4} \operatorname{mult}_{\pi\left(M_{i}\right)}(H) \operatorname{mult}_{\pi\left(M_{i}\right)}(G) \geq 4 m+2 \operatorname{mult}_{q}(G)
$$

shows that the maximum value $v_{m}$ can be attained when the image of the injective map $\iota_{m}$ in (4.2) is contained in the set of the monomials corresponding to the integral points in the shade area of the following diagram:

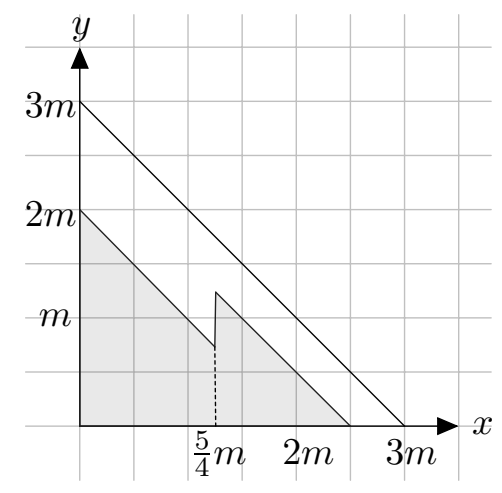

To be precise, the monomials of the set $\mathcal{C}_{m}$ correspond to the integral points below and on the line joining $(2 m, 0)$ and $(0,2 m)$. The images $\iota_{m}\left(u_{1}\right), \ldots, \iota_{m}\left(u_{a}\right)$ sit in the shade area above the line joining $(2 m, 0)$ and $(0,2 m)$.

Then, Figure 1 shows that

$$
\limsup _{m} \frac{m \ell_{m}}{v_{m}}=\frac{15}{14} .
$$

Case 2. There is a divisor $C$ in $\left|-K_{S}\right|$ that has multiplicity 3 at $p$.

The curve $\pi(C)$ consists of three lines $L_{1}, L_{2}, L_{3}$ passing through the point $q$. We may assume that the points $\pi\left(M_{1}\right)$ and $\pi\left(M_{2}\right)$ belong to $L_{1}$. Then the points $\pi\left(M_{3}\right)$ and $\pi\left(M_{4}\right)$ belong to the union of $L_{2}$ and $L_{3}$. Furthermore, we may assume that $L_{1}$ is defined by $x=0, L_{2}$ by $y=0$ and $L_{3}$ by $x+y=0$. Then

$$
t_{1}=1, t_{2}=x, t_{3}=y, t_{4}=x^{2}, t_{5}=x y, t_{6}=x y(x+y)
$$

form a basis for $\mathcal{T}_{1}$. We may take

$$
\mathbf{x}_{1,1}=1, \mathbf{x}_{1,2}=x, \mathbf{x}_{1,3}=y, \mathbf{x}_{1,4}=x^{2}, \mathbf{x}_{1,5}=x y, \mathbf{x}_{1,6}=x y^{2} .
$$


The set $\mathcal{C}_{m}$ consists of the monomials corresponding the integral points in the shade area of the following diagram:

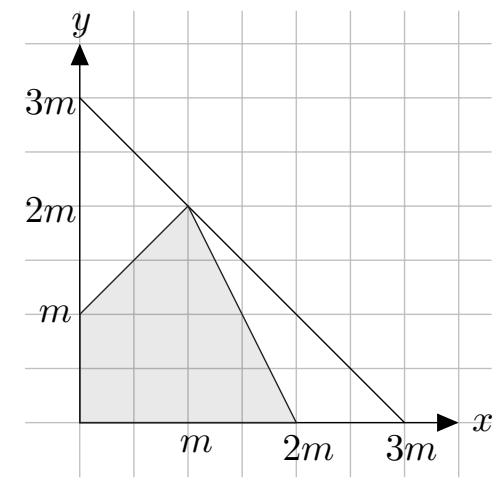

Note that the number of monomials in this set is exactly $\ell_{m}$. Therefore, $a=0$ in Step 2 and Figure 1 shows that

$$
\limsup _{m} \frac{m \ell_{m}}{v_{m}}=\frac{15}{13}
$$

Cases 1 and 2 complete the proof.

\section{K-Semistable del Pezzo surfaces II}

In this section, together with Section 3, we complete the proof of the second statement of Main Theorem.

Let $S$ be the del Pezzo surface of degree 6 . This surface can be obtained by blowing up $\mathbb{P}^{2}$ at $p_{1}=[0: 0: 1], p_{2}=[0: 1: 0]$ and $p_{3}=[1: 0: 0]$. Let $\phi_{3}: S \rightarrow \mathbb{P}^{2}$ be the blow-up with the exceptional curves $E, F, G$. For a fixed positive integer $m$, the space $\mathrm{H}^{0}\left(S, \mathcal{O}_{S}\left(-m K_{S}\right)\right)$ can be regarded as the subspace $\mathcal{L}_{m}$ of $\mathrm{H}^{0}\left(\mathbb{P}^{2}, \mathcal{O}_{\mathbb{P}^{2}}(3 m)\right)$ consisting of the sections vanishing at $p_{1}, p_{2}, p_{3}$ with order at least $m$. The set

$$
\mathcal{B}_{3}=\left\{x^{a} y^{b} z^{c} \mid a, b, c \text { are integers with } a+b+c=3 m \text { and } 0 \leq a, b, c \leq 2 m\right\}
$$

forms a basis for $\mathcal{L}_{m}$. Let $D_{a, b, c}$ be the divisor defined by $x^{a} y^{b} z^{c}$ on $\mathbb{P}^{2}$. Then the divisor

$$
D=\frac{1}{m\left(3 m^{2}+3 m+1\right)} \sum_{x^{a} y^{b} z^{c} \in \mathcal{B}_{3}}\left(\phi_{3}^{*}\left(D_{a, b, c}\right)-m E-m F-m G\right)
$$

is an anticanonical $\mathbb{Q}$-divisor of $m$-basis type on $S$. We immediately see that

$$
D=L_{x}+L_{y}+L_{y}+E+F+G,
$$

where $L_{x}, L_{y}, L_{z}$ are the proper transforms of the lines on $\mathbb{P}^{2}$ defined by $x=0, y=0, z=0$, respectively. Therefore, $\delta(S) \leq 1$.

Theorem 5.1. The $\delta$-invariant of the del Pezzo surface of degree 6 is 1 .

Proof. Fix a positive integer $m$ and set $\ell_{m}=h^{0}\left(S, \mathcal{O}_{S}\left(-m K_{S}\right)\right)$. Let $\left\{s_{1}, \ldots, s_{\ell_{m}}\right\}$ be a basis of $\mathrm{H}^{0}\left(S, \mathcal{O}_{S}\left(-m K_{S}\right)\right)$. We denote the effective divisor of the section $s_{i}$ by $D_{i}$. Put $D=\sum D_{i}$. Let $p$ be an arbitrary point on $S$.

Case 1. The point $p$ is not an intersection point of two (-1)-curves.

In this case, there is a birational morphism $\pi: S \rightarrow \mathbb{P}^{2}$ that is an isomorphism around the point $p$. We can use the exactly same method as in the previous section. In order to apply the same method, we set $q=\pi(p)$ and use the same notations as before. By a suitable coordinate change, we assume that $q=[0: 0: 1]$. 
Subcase 1. The point $p$ does not lie on any (-1)-curve.

Note that $\operatorname{dim}_{k} \mathcal{T}_{1}=7$. Since the three lines determined by the points $q$ and $\pi\left(M_{i}\right), i=1,2,3$, are distinct, by a suitable coordinate change we may assume that

$$
t_{1}=1, t_{2}=x, t_{3}=y, t_{4}=x^{2}, t_{5}=x y, t_{6}=y^{2}, t_{7}=x y(x+y)
$$

form a basis for $\mathcal{T}_{1}$. We may therefore take

$$
\mathbf{x}_{1,1}=1, \mathbf{x}_{1,2}=x, \mathbf{x}_{1,3}=y, \mathbf{x}_{1,4}=x^{2}, \mathbf{x}_{1,5}=x y, \mathbf{x}_{1,6}=y^{2}, \mathbf{x}_{1,7}=x^{2} y .
$$

Then the set $\mathcal{C}_{m}$ consists of the monomials corresponding to the integral points in the shade area of the following diagram:

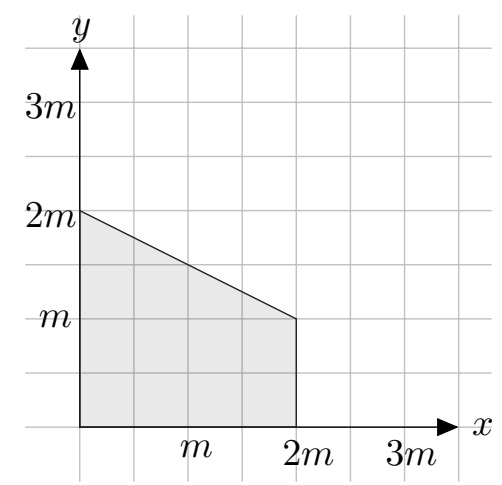

Note that the number of monomials in this set is exactly $\ell_{m}$. Therefore, $a=0$ in Step 2 and Figure 1 shows that

$$
\limsup _{m} \frac{m \ell_{m}}{v_{m}}=\frac{9}{8}
$$

Subcase 2. The point p lies on a single (-1)-curve.

In this case, by a suitable coordinate change we may assume that

$$
t_{1}=1, t_{2}=x, t_{3}=y, t_{4}=x^{2}, t_{5}=x y, t_{6}=x^{2} y, t_{7}=x y^{2}
$$

form a basis for $\mathcal{T}_{1}$. Then

$$
\mathbf{x}_{1,1}=1, \mathbf{x}_{1,2}=x, \mathbf{x}_{1,3}=y, \mathbf{x}_{1,4}=x^{2}, \mathbf{x}_{1,5}=x y, \mathbf{x}_{1,6}=x^{2} y, \mathbf{x}_{1,7}=x y^{2} .
$$

The set $\mathcal{C}_{m}$ consists of the monomials corresponding to the integral points in the shade area of the following diagram:

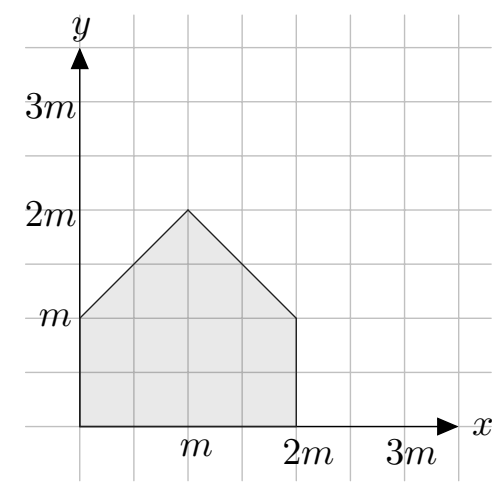


The number of monomials in this set is exactly $\ell_{m}$. Therefore, $a=0$ in Step 2 and Figure 1 shows that

$$
\limsup _{m} \frac{m \ell_{m}}{v_{m}}=1
$$

Case 2. The point $p$ is an intersection point of two (-1)-curves.

There is a birational morphism $\phi: S \rightarrow \mathbb{P}^{1} \times \mathbb{P}^{1}$ that is an isomorphism around the point $p$. The morphism $\phi$ is obtained by contracting two suitable disjoint (-1)-curves $M_{1}, M_{2}$ on $S$.

For an effective divisor $C \in\left|-m K_{S}\right|$, the divisor $\phi(C)$ is a curve of bidegree $(2 m, 2 m)$ on $\mathbb{P}^{1} \times \mathbb{P}^{1}$ which passes through the points $\phi\left(M_{1}\right)$ and $\phi\left(M_{2}\right)$ with multiplicities at least $m$. Such divisors yield an $\ell_{m}$-dimensional subspace of $\mathrm{H}^{0}\left(\mathbb{P}^{1} \times \mathbb{P}^{1}, \mathcal{O}_{\mathbb{P}^{1} \times \mathbb{P}^{1}}\left(-m K_{\mathbb{P}^{1} \times \mathbb{P}^{1}}\right)\right)$. The effective divisors $\phi\left(D_{1}\right), \ldots, \phi\left(D_{\ell_{m}}\right)$ induce a basis for this subspace.

Let $G$ be an effective divisor on $\mathbb{P}^{1} \times \mathbb{P}^{1}$ that comes from $\left|-m K_{S}\right|$. Let $L_{1}$ and $L_{2}$ be the two 0 -curves that pass through the point $\phi(p)$. We write $G=m_{1} L_{1}+m_{2} L_{2}+\Omega$, where $m_{1}$ and $m_{2}$ are non-negative integers and $\Omega$ is an effective divisor whose support contains neither $L_{1}$ nor $L_{2}$. We may assume that $\phi\left(M_{1}\right)$ belongs to $L_{1}$ and $\phi\left(M_{2}\right)$ belongs to $L_{2}$.

We use a bihomogeneous coordinate system $([x: u],[y: v])$ for $\mathbb{P}^{1} \times \mathbb{P}^{1}$. For the proof, we may assume that $\phi(p)=([0: 1],[0: 1])$. Putting $u=1$ and $v=1$, we may regard $x$ and $y$ as local coordinates around the point $\phi(p)$. Even though we maps the surface $S$ onto $\mathbb{P}^{1} \times \mathbb{P}^{1}$ instead of $\mathbb{P}^{2}$, the original method to estimate $c_{p}\left(S, \frac{1}{m \ell_{m}} D\right)$ works verbatim for this case.

The inequalities

$$
\begin{aligned}
& 2 m=L_{1} \cdot\left(m_{1} L_{1}+m_{2} L_{2}+\Omega\right) \geq m_{2}+\left(m-m_{1}\right)+\operatorname{mult}_{\phi(p)}(\Omega), \\
& 2 m=L_{2} \cdot\left(m_{1} L_{1}+m_{2} L_{2}+\Omega\right) \geq m_{1}+\left(m-m_{2}\right)+\operatorname{mult}_{\phi(p)}(\Omega)
\end{aligned}
$$

imply $\left|m_{1}-m_{2}\right| \leq m$ and

$$
m-\left|m_{1}-m_{2}\right| \geq \operatorname{mult}_{\phi}(p)(\Omega) .
$$

Note that $0 \leq m_{1}, m_{2} \leq 2 m$. This implies that the image of the injective map $\iota_{m}$ in (4.2) can be arranged to be contained in the set of the monomials corresponding to the integral points in the shade area of the following diagram:

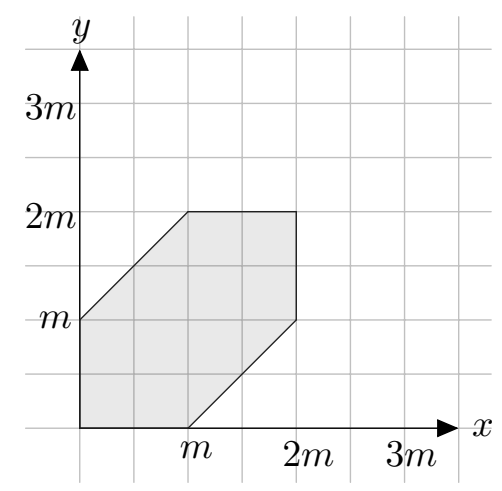

Note that the number of monomials in this set is exactly $\ell_{m}$. Figure 1 shows that

$$
\limsup _{m} \frac{m \ell_{m}}{v_{m}}=1
$$

From Cases 1 and 2, we can draw the conclusion that $\delta(S)=1$. 
Now we consider the space $\mathbb{P}^{1} \times \mathbb{P}^{1}$. We use a bihomogeneous coordinate system $([x: u],[y: v])$ for $\mathbb{P}^{1} \times \mathbb{P}^{1}$. As before, fix a positive integer $m$ and set $\ell_{m}=h^{0}\left(\mathbb{P}^{1} \times \mathbb{P}^{1}, \mathcal{O}_{\mathbb{P}^{1} \times \mathbb{P}^{1}}\left(-m K_{\mathbb{P}^{1} \times \mathbb{P}^{1}}\right)\right)$. The $\ell_{m}$ monomials of bidegree $(2 m, 2 m)$ in variables $x, u ; y, v$ form a basis for $\mathrm{H}^{0}\left(\mathbb{P}^{1} \times\right.$ $\left.\mathbb{P}^{1}, \mathcal{O}_{\mathbb{P}^{1} \times \mathbb{P}^{1}}\left(-m K_{\mathbb{P}^{1} \times \mathbb{P}^{1}}\right)\right)$. Therefore, the devisor defined by $x u y v=0$ is an anticanonical divisor of $m$-basis type. This shows

$$
\delta\left(\mathbb{P}^{1} \times \mathbb{P}^{1}\right) \leq 1
$$

Theorem 5.2. The $\delta$-invariant of $\mathbb{P}^{1} \times \mathbb{P}^{1}$ is 1 .

Proof. Let $\left\{s_{1}, \ldots, s_{\ell_{m}}\right\}$ be a basis of the space $\mathrm{H}^{0}\left(\mathbb{P}^{1} \times \mathbb{P}^{1}, \mathcal{O}_{\mathbb{P}^{1} \times \mathbb{P}^{1}}\left(-m K_{\mathbb{P}^{1} \times \mathbb{P}^{1}}\right)\right)$. Denote the effective divisor defined by the section $s_{i}$ by $D_{i}$. Set $D=\sum D_{i}$. For a given point $p \in \mathbb{P}^{1} \times \mathbb{P}^{1}$, as before, the inequality

$$
c_{p}\left(\mathbb{P}^{1} \times \mathbb{P}^{1}, D\right) \geq \frac{1}{m \ell_{m}}
$$

will be verified.

We may assume that $p=([0: 1],[0: 1])$. Putting $u=1$ and $v=1$, we may regard $x$ and $y$ as local coordinates around the point $p$. As Case 2 in Theorem 5.1 , the original method to estimate $c_{p}\left(\mathbb{P}^{1} \times \mathbb{P}^{1}, \frac{1}{m \ell_{m}} D\right)$ works verbatim.

Let $G$ be an effective divisor in $\left|-m K_{\mathbb{P}^{1} \times \mathbb{P}^{1}}\right|$. Let $L_{1}$ and $L_{2}$ be the two 0 -curves that pass through the point $p$. We write $G=m_{1} L_{1}+m_{2} L_{2}+\Omega$, where $m_{1}$ and $m_{2}$ are non-negative integers and $\Omega$ is an effective divisor whose support contains neither $L_{1}$ nor $L_{2}$.

The inequalities

$$
\begin{aligned}
& 2 m=L_{1} \cdot\left(m_{1} L_{1}+m_{2} L_{2}+\Omega\right) \geq m_{2}+\operatorname{mult}_{p}(\Omega), \\
& 2 m=L_{2} \cdot\left(m_{1} L_{1}+m_{2} L_{2}+\Omega\right) \geq m_{1}+\operatorname{mult}_{p}(\Omega)
\end{aligned}
$$

imply $0 \leq m_{1}, m_{2} \leq 2 m$ and

$$
2 m-\max \left\{m_{1}, m_{2}\right\} \geq \operatorname{mult}_{p}(\Omega) .
$$

This implies that the image of the injective map $\iota_{m}$ in (4.2) is contained in the set of the monomials corresponding to the integral points in the shade area of the following diagram:

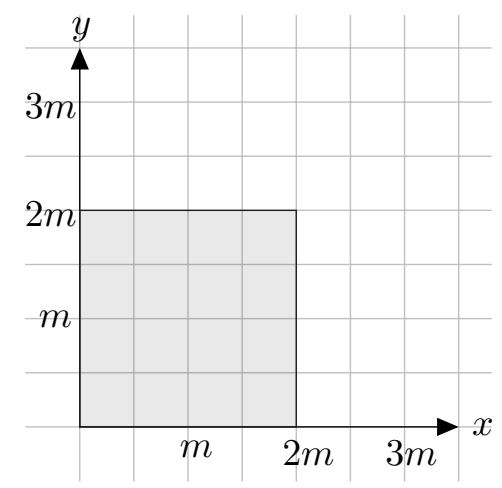

Figure 1 then shows that

$$
\limsup _{m} \frac{m \ell_{m}}{v_{m}}=1
$$




\section{Non-K-Semistable Del Pezzo surfaces}

In this section, we verify the last statement of Main Theorem. Since Conjecture 1.7 has not been verified completely, at this moment we cannot say that the last statement of Main Theorem implies that the Hirzebruch surface $\mathbb{F}_{1}$ and the del Pezzo surface of degree 7 are not K-semistable. However, it is able to serve as a good evidence for Conjecture 1.7.

Let $[x: y: z]$ be a homogeneous coordinate for $\mathbb{P}^{2}$. The Hirzebruch surface $\mathbb{F}_{1}$ can be obtained by blowing up $\mathbb{P}^{2}$ at $p_{1}=[0: 0: 1]$. Let $\phi_{1}: \mathbb{F}_{1} \rightarrow \mathbb{P}^{2}$ be the blow-up with the exceptional divisor $E$. For a fixed positive integer $m$, the space $\mathrm{H}^{0}\left(\mathbb{F}_{1}, \mathcal{O}_{\mathbb{F}_{1}}\left(-m K_{\mathbb{F}_{1}}\right)\right)$ can be regarded as the subspace $\mathcal{M}_{1}$ of $\mathrm{H}^{0}\left(\mathbb{P}^{2}, \mathcal{O}_{\mathbb{P}^{2}}(3 m)\right)$ consisting of the sections vanishing at $p_{1}$ with order at least $m$. The set

$$
\mathcal{B}_{1}=\left\{x^{a} y^{b} z^{c} \mid a, b, c \text { are non-negative integers with } a+b+c=3 m, c \leq 2 m\right\}
$$

forms a basis for $\mathcal{M}_{1}$. Let $D_{a, b, c}$ be the divisor defined by $x^{a} y^{b} z^{c}$ on $\mathbb{P}^{2}$. Then the divisor

$$
D=\frac{1}{m(2 m+1)^{2}} \sum_{x^{a} y^{b} z^{c} \in \mathcal{B}_{1}}\left(\phi_{1}^{*}\left(D_{a, b, c}\right)-m E\right)
$$

is an anticanonical $\mathbb{Q}$-divisor of $m$-basis type on $\mathbb{F}_{1}$. The multiplicity of $D$ along the curve $E$ is $\frac{7 m+4}{6 m+3}$. Therefore,

$$
\delta_{m}\left(\mathbb{F}_{1}\right) \leq \frac{6 m+3}{7 m+4}
$$

and hence

$$
\delta\left(\mathbb{F}_{1}\right) \leq \frac{6}{7}
$$

We now let $S$ be the del Pezzo surface of degree 7 . It can be obtained by blowing up $\mathbb{P}^{2}$ at $p_{1}=[0: 0: 1]$ and $p_{2}=[0: 1: 0]$. Let $\phi_{2}: S \rightarrow \mathbb{P}^{2}$ be the blow-up with the exceptional curves $E$ and $F$. For a fixed positive integer $m$, the space $\mathrm{H}^{0}\left(S, \mathcal{O}_{S}\left(-m K_{S}\right)\right)$ can be regarded as the subspace $\mathcal{M}_{2}$ of $\mathrm{H}^{0}\left(\mathbb{P}^{2}, \mathcal{O}_{\mathbb{P}^{2}}(3 m)\right)$ consisting of the sections vanishing at $p_{1}$ and $p_{2}$ with order at least $m$. The set

$$
\mathcal{B}_{2}=\left\{x^{a} y^{b} z^{c} \mid a, b, c \text { are non-negative integers with } a+b+c=3 m, b \leq 2 m, c \leq 2 m\right\}
$$

forms a basis for $\mathcal{M}_{2}$. Let $C_{a, b, c}$ be the divisor defined by $x^{a} y^{b} z^{c}$ on $\mathbb{P}^{2}$. The anticanonical $\mathbb{Q}$-divisor

$$
C=\frac{2}{7 m^{2}(m+1)+2 m} \sum_{x^{a} y^{b} z^{c} \in \mathcal{B}_{2}}\left(\phi_{2}^{*}\left(C_{a, b, c}\right)-m E-m F\right)
$$

is of $m$-basis type on $S$. The multiplicity of $C$ along the proper transform of the curve defined by $x=0$ is $\frac{25 m^{2}+27 m+8}{21 m(m+1)+6}$. Therefore,

$$
\delta_{m}(S) \leq \frac{21 m(m+1)+6}{25 m^{2}+27 m+8},
$$

and hence

$$
\delta(S) \leq \frac{21}{25}
$$




\section{APPENDIX}

In Appendix Lemmas 4.1, 4.3 and 4.6 are verified. All the notations are the same as those in the beginning of Section 4 .

We consider the vector space

$$
V_{\lambda}:=\bigoplus_{n=0}^{\lambda} k[x, y]_{n}
$$

with an ordered basis $\left\{x^{\alpha} y^{\beta} \mid \alpha+\beta \leq \lambda\right\}$, where $k[x, y]_{n}$ is the $(n+1)$-dimensional vector space of homogeneous polynomials of degree $n$ in variables $x, y$. The order of the basis is given in the following way:

(1) the graded lexicographic order with $x \prec y$ except for Case 2 in Theorem 4.7 and Case 2 in Theorem 4.11:

(2) the graded lexicographic order with $y \prec x$ for Case 2 in Theorem 4.11,

(3) the order for Case 2 in Theorem 4.7 satisfies the properties:

(a) $x^{\alpha_{1}} y^{\beta_{1}} \prec x^{\alpha_{2}} y^{\beta_{2}}$ if $\alpha_{1}+\beta_{1}<\alpha_{2}+\beta_{2}$;

(b) $x^{\alpha} y^{\alpha} \prec x^{\alpha_{2}} y^{\beta_{2}}$ if $\alpha_{2} \neq \beta_{2}$ and $\alpha_{2}+\beta_{2}=2 \alpha$.

Note that these orders make the monomial $\prod_{i=1}^{d+1} \mathbf{x}_{1, i}^{n_{i}}$ in $\mathcal{C}_{m}$ smaller than any other monomials that appear in $\prod_{i=1}^{d+1} t_{i}^{n_{i}}$.

Since $f_{m, i}$ is a member of the vector space $V_{3 m}$, we may express the polynomial $f_{m, i}$ as a $1 \times \sigma$ matrix with respect to the given ordered basis, where $\sigma=\frac{(3 m+1)(3 m+2)}{2}$. By writing these $1 \times \sigma$ matrices as rows, we can express the $\ell_{m}$ polynomials $f_{m, 1}, \cdots, f_{m, \ell_{m}}$ altogether as a single $\ell_{m} \times \sigma$ matrix $M_{F}$. Since $f_{m, 1}, \cdots, f_{m, \ell_{m}}$ are linearly independent, the rank of the matrix $M_{F}$ is exactly $\ell_{m}$.

Let $E$ be a row echelon form of the matrix $M_{F}$. Then there is an $\ell_{m} \times \ell_{m}$ invertible matrix $T$ such that $M_{F}=T E$. Since the rank of $M_{F}$ is $\ell_{m}$, the matrix $E$ does not have any zero row. The $i$-th row of $E$ represents a polynomial $h_{m, i}$ that belongs to $\mathcal{L}_{m}$. Its Zariski tangent term $t_{m, i}$ is represented by the pivot (the first non-zero entry from the left in a row) and the entries whose corresponding monomials have the same degree as the monomial corresponding to the pivot. In particular, the Zariski tangent term contains the monomial corresponding to the pivot. The polynomials $h_{m, i}$ form a basis for the space $\mathcal{L}_{m}$ and their Zariski tangent terms $t_{m, i}$ form a basis for the space $\mathcal{T}_{m}$.

Since the monomial $\prod_{i=1}^{d+1} \mathbf{x}_{1, i}^{n_{i}}$ in $\mathcal{C}_{m}$ is smaller than any other monomials that appear in $\prod_{i=1}^{d+1} t_{i}^{n_{i}}$, the set of the monomials corresponding to the columns with the pivots of $E$ must contain the set $\mathcal{C}_{m}$.

By collecting the $\ell_{m}$ pivot columns of $E$ in order, we obtain an $\ell_{m} \times \ell_{m}$ upper triangular matrix with the pivots on the diagonal. Denote this minor matrix of $E$ by $\widetilde{E}$. We also denote the $\ell_{m} \times \ell_{m}$ matrix $T \widetilde{E}$ by $\widetilde{M}_{F}$. The entries of $i$-th row of $\widetilde{M}_{F}$ are the coefficients of the monomials in $f_{m, i}$ corresponding to the pivot columns of $E$. Since the matrix $\widetilde{M}_{F}$ is nonsingular, we can choose a single non-zero entry from each column of $\widetilde{M}_{F}$ in such a way that the non-zero entries are selected exactly one time from each row. This proves Lemmas 4.1 and 4.3 .

For Lemma 4.6. we consider the vector space $V_{\lambda}$ with a sufficiently large positive integer $\lambda$ so that we could write polynomials of bigger degrees as matrices. Note that the change of coordinate $x+A_{1} y^{\beta} \mapsto x$ in Step $\mathrm{C}$ is given with $\beta>1$. Since

$$
f_{m, i}(x, y)=\sum_{j=1}^{\ell_{m}} T_{i j} h_{m, j}(x, y)
$$


for each $i$, where $T_{i j}$ is the entry of $T$ in the $i$-th row and the $j$-th column, we have

$$
f_{m, i}^{(1)}(x, y)=f_{m, i}\left(x-A_{1} y^{\beta}, y\right)=\sum_{j=1}^{\ell_{m}} T_{i j} h_{m, j}\left(x-A_{1} y^{\beta}, y\right) .
$$

Since $\beta>1$, we immediately see that the change of coordinate does not give any effect on the Zariski tangent term of $h_{m, i}$ at all. It therefore leaves the positions of the pivot columns of $E$ unchanged. Therefore, by the same argument as for Lemmas 4.1 and 4.3 , we can obtain an injection in Lemma 4.6. Furthermore, the same argument works inductively for $\left\{f_{m, i}^{(k)}\right\}, k>1$. This completes the proof of Lemma 4.6 .

\section{REFERENCES}

[1] Cheltsov, I.: Log canonical thresholds of del Pezzo surfaces. Geom. Funct. Anal. 11, 1118-1144 (2008)

[2] Cheltsov, I., Park, J.: Global log-canonical thresholds and generalized Eckardt points. Sb. Math. 193 (5-6), 779-789 (2002)

[3] Cheltsov, I., Park, J.: Sextic double solids. In: Cohomological and geometric approaches to rationality problems, Progr. Math., 282, Birkhäuser, Boston, MA, 75-132 (2010)

[4] Cheltsov, I., Park, J., Won. J: Log canonical thresholds of certain Fano hypersurfaces. Math. Z. 276 (1-2), 51-79 (2014)

[5] Cheltsov, I., Shramov, K.: Log-canonical thresholds for nonsingular Fano threefolds. With an appendix by J.-P. Demailly. Russian Math. Surveys 63 (5), 859-958 (2008)

[6] Chen, X., Donaldson, S., Sun, S.: Kähler-Einstein metrics on Fano manifolds I: approximation of metrics with cone singularities. J. Amer. Math. Soc. 28 (1), 183-197 (2015)

[7] Chen, X., Donaldson, S., Sun, S.: Kähler-Einstein metrics on Fano manifolds, II: limits with cone angle less than $2 \pi$. J. Amer. Math. Soc. 28 (1), 199-234 (2015)

[8] Chen, X., Donaldson, S., Sun, S.: Kähler-Einstein metrics on Fano manifolds III: limits as cone angle approaches $2 \pi$ and completion of the main proof. J. Amer. Math. Soc. 28 (1), 235-278 (2015)

[9] Demailly, P., Kollár, J.: Semicontinuity of complex singularity exponents and Kähler-Einstein metrics on Fano orbifolds. Ann. Ec. Norm. Sup 34, 525-556 (2001)

[10] Fujita, K.: On K-stability and the volume functions of $\mathbb{Q}$-Fano varieties. Proc. Lond. Math. Soc. 113 (5), 541-582 (2016)

[11] Fujita, K.: K-stability of Fano manifolds with not small alpha invariants. preprint arXiv:1606.08261

[12] Fujita, K.: On Berman-Gibbs stability and K-stability of $\mathbb{Q}$-Fano varieties. Compositio Math. 152, 288-298 (2016)

[13] Fujita, K., Odaka, Yu.: On the K-stability of Fano varieties and anticanonical divisors. preprint arXiv:1602.01305

[14] Hacking, P., Prokhorov, Yu.: Smoothable del Pezzo surfaces with quotient singularities. Compositio Math. 146 (1), 169-192 (2010)

[15] Hwang, J.-M., Kim, H., Lee, Y., Park, J.: Slopes of smooth curves on Fano manifolds. Bull. Lond. Math. Soc. 43 (5), 827-839 (2011)

[16] Kempf, G.: Instability in invariant theory. Ann. of Math. (2) 108 (2), 299-316 (1978)

[17] Kollár, J.: Singularities of pairs. In: Algebraic geometry (Santa Cruz, 1995) Part 1, Proc. Sympos. Pure Math., 62, Amer. Math. Soc., 221-287 (1997)

[18] Manetti, M.: Normal degenerations of the complex projective plane. J. Reine Angew. Math. 419, 89-118 (1991)

[19] Manetti, M.: Normal projective surfaces with $\rho=1, P_{-1} \geq 5$. Rend. Sem. Mat. Univ. Padova 89, 195-205 (1993)

[20] Odaka, Yu., Sano, Yu.: Alpha invariant and K-stability of $\mathbb{Q}$-Fano varieties. Adv. Math. 229 (5), 2818-2834 (2012)

[21] Panov, D., Ross, J.: Slope stability and exceptional divisors of high genus. Math. Ann. 343 (1), 79-101 (2009)

[22] Prokhorov, Yu.: A note on degenerations of del Pezzo surfaces. Ann. Inst. Fourier (Grenoble) 65 (1), 369-388 (2015)

[23] Ross, J., Thomas, R.: A study of the Hilbert-Mumford criterion for the stability of projective varieties. J. Algebraic Geom. 16 (2), 201-255 (2007)

[24] Tian, G.: On Kähler-Einstein metrics on certain Kähler manifolds with $c_{1}(M)>0$. Invent. Math. 89, 225-246 (1987) 
[25] Tian, G.: K-stability and Kähler-Einstein metrics. Comm. Pure Appl. Math. 68 (7), $1085-1156$ (2015). Corrigendum: K-Stability and Kähler-Einstein Metrics. 68 (11), 2082-2083 (2015)

Jihun Park

Center for Geometry and Physics, Institute for Basic Science (IBS)

77 Cheongam-ro, Nam-gu, Pohang, Gyeongbuk, 37673, Korea.

Department of Mathematics, POSTECH

77 Cheongam-ro, Nam-gu, Pohang, Gyeongbuk, 37673, Korea.

wlog@postech.ac.kr

Joonyeong Won

Center for Geometry and Physics, Institute for Basic Science (IBS)

77 Cheongam-ro, Nam-gu, Pohang, Gyeongbuk, 37673, Korea.

leonwon@kias.re.kr 